\title{
Dietary agrobiodiversity for improved nutrition and health outcomes within a transitioning indigenous Solomon Island food system
}

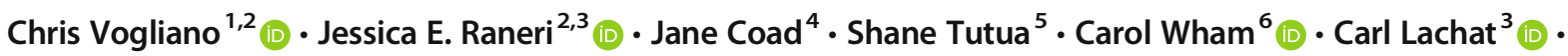 \\ Barbara Burlingame ${ }^{1}$ (B)
}

Received: 4 September 2019 / Accepted: 6 April 2021 / Published online: 10 May 2021

(C) The Author(s) 2021

\begin{abstract}
Indigenous food systems of Pacific Small Island Developing Countries contain vast biological and cultural diversity. However, a nutrition transition is underway, characterized by shifts away from traditional diets in favour of imported and modern foods, contributing to some of the highest rates of obesity and Diabetes Type 2 Mellitus in the world. Using a mixed method approach, this study aimed to assess dietary agrobiodiversity's relationship with nutrition indicators related to diet quality and anthropometrics within the context of the rural and Indigenous food system of Baniata village, located in the Western Province of Solomon Islands (Melanesia). A secondary aim was to evaluate the contribution of agrobiodiversity from the local food system to diet quality. A comprehensive nutrition survey was administered to the women primarily responsible for cooking of randomly selected households $(n=30)$. Additionally, 14 participatory focus group discussions captured the historical narrative of food system transitions, were hosted over a period of seven days, and included men, women and youth. Dietary intakes of the participants were reported below the estimated average requirement (EAR) for several essential nutrients, including protein (53\%), calcium (96.6\%), vitamin B1 (86.6\%), vitamin B2 (80\%), vitamin A (80\%), zinc (40\%) and fibre (77\%). Focus group participants built a timeline of key historical and climatic transitions perceived to be drivers of dietary shifts away from traditional foods and towards imported and processed foods. Participants identified 221 species and varieties of agrobiodiverse foods available for cultivation or wild collection. Based on $24 \mathrm{~h}$ diet recalls, 87 were found to be utilised. Participants who consumed foods of a wider diversity of species richness had a higher probability of achieving recommended nutrition intakes and a lower body fat percentage $\left(r^{2}=0.205 ; p=0.012\right)$. Our results suggest a nutrition transition is underway, and strategies harnessing traditional knowledge of nutrient-dense, agrobiodiverse foods can help improve food and nutrition security.
\end{abstract}

Keywords Sustainable diets $\cdot$ Food security $\cdot$ Wild foods $\cdot$ Pacific Islands $\cdot$ Melanesia $\cdot$ Diet quality

Chris Vogliano

chrisvogliano@gmail.com

1 School of Health Sciences, Massey University, Wallace St., Wellington 6021, New Zealand

2 Bioversity International, Healthy Diets from Sustainable Food Systems Initiative, Sydney, Australia

3 Department of Food Technology, Safety and Health, Ghent University, Ghent, Belgium

4 School of Food and Advanced Technology, Massey University, Manawatū campus, Palmerston North, New Zealand

5 Zai Na Tina Centre for Organic Systems, Honiara, Solomon Islands

6 School of Sport, Exercise and Nutrition, Massey University, Albany Campus, Auckland, New Zealand

\section{Introduction}

Pacific Small Island Developing States (PSIDS) are experiencing disproportionate threats to their food and nutrition security through rising rates of malnutrition and increased vulnerability to climate change (Allen, 2015; Haddad et al., 2015; Hughes \& Lawrence, 2005). The sustainability of Indigenous food systems are particularly at risk from both increased consumption of nutrient-poor, imported foods (McIver et al., 2016; Shrimpton et al., 2016) and rapidly changing weather patterns (Allen, 2015; Warrick et al., 2017). Climate-related events, such as stronger and more frequent storms, heavier rains, longer droughts, and rising ocean temperatures all add pressures to the already stressed food systems. Climate change is projected to magnify all forms of malnutrition (Phalkey et al., 2015). The future of habitation 
and agricultural production in PSIDS relies on adaptation to the adverse impacts of climate change and malnutrition. To date, insufficient research exists examining the role between food production systems, agrobiodiversity and malnutrition in PSIDS (Haynes et al., 2018).

Sustainable Development Goal (SDG 2 (Zero Hunger) is focused on eliminating all forms of malnutrition through sustainable diets. Sustainable diets are increasingly seen as a precondition for assuring food security (Berry et al., 2015; Canavan et al., 2017; Smith \& Gregory, 2013). Sustainable diets are defined as being protective and respectful of biodiversity and ecosystems, culturally acceptable, accessible, economically fair and affordable, nutritionally adequate, safe and healthy while optimizing natural and human resources (Burlingame \& Dernini, 2012). Achieving SDG 2 requires multifaceted coordination between agricultural, nutrition and health sectors, all encouraging sustainable food systems for current and future generations, particularly within the scope of climate change.

Transitioning foods systems characterized by a shift away from local and traditional foods towards globalised food systems is eliciting a rise in food and nutrition insecurity and noncommunicable diseases (NCDs) across many PSIDS (Foley et al., 2011). Increasing reliance on imported and ultraprocessed foods, which are typically nutrient-poor and calorie-dense, is a leading driver of malnutrition and contribute to NCDs (Hughes \& Lawrence, 2005; Thow et al., 2011). Furthermore, as global anthropogenic $\mathrm{CO}_{2}$ emissions continue to rise (with negligible contribution from those living in PSIDS), staple crops are projected to decrease in micronutrient density, adding increased nutritional vulnerability to women and children (Smith \& Myers, 2018). Reliance on a smaller diversity of foods (diet homogenization) is creating conditions for poor diet quality and micronutrient inadequacies, as well as an increased susceptibility towards climate change (Sandifer et al., 2015).

Agricultural biodiversity, or agrobiodiversity, is a key component to ensuring healthy and sustainable food systems (Zimmerer et al., 2019). Agrobiodiversity includes all components of biological diversity related to food, agriculture, food culture, and related ecosystem services and is being lost at an alarming rate. Since the 1900 s, nearly $75 \%$ of plant genetic diversity has been lost as farmers worldwide have left their multiple local varieties and landraces for genetically uniform, high-yielding varieties (Rischkowsky \& Pilling, 2007). Today, only 12 species of plants and 5 species of animals make up $75 \%$ of the world's food supply (Convention on Biological Diversity, FAO, The World Bank, UNEP, and UNDP, 2015). Transitioning diets and eroding cultural traditions in PSIDS threaten local agrobiodiversity and subsequently, the resiliency of local Indigenous food systems.

Neglected and underutilised species (NUS), one key component of agrobiodiversity, are gaining global attention as a potential solution towards sustainable food systems and have implications for providing locally available, culturally acceptable, nutritious, and cost-effective foods for vulnerable populations (Baldermann et al., 2016; Ebert, 2014; Raneri et al., 2019). NUS foods are often nutrient-dense, productive, ecologically-adapted and socio-economically accepted (Li \& Siddique, 2018). For instance, researchers have identified some nutrient-rich NUS in PSIDS, such as the Fe'i banana (Musa $\times$ troglodytarum L.), a bright orange, carotenoid-rich species with 100 times the $\beta$-carotene content of the common Cavendish banana $(2230 \mu \mathrm{g} / 100 \mathrm{~g}$ vs. $21 \mu \mathrm{g} / 100 \mathrm{~g})$ (Buah et al., 2016). Improved understanding of agrobiodiversity in PSIDS can help guide sustainable agricultural diversification and intensification strategies aimed at achieving SDG 2.

\subsection{Solomon Island food systems}

Solomon Islands is an archipelago country made up of over 900 islands with a rich cultural and agricultural history. Indigenous Solomon Islanders (pop. $n=612,000$ ) have traditionally relied on locally grown, biodiverse foods as their primary sources for food and nutrition. However, together with the wider Pacific region, they are now experiencing food system and nutrition transitions, and subsequent adverse impacts of changing diets (Santos et al., 2019; Sievert et al., 2019). Top imported items in 2016 include white rice $(>43.2 \mathrm{k}$ tonnes), wheat ( $>16.8 \mathrm{k}$ tonnes), sugar ( $>6.5 \mathrm{k}$ tonnes), and processed foods ( $>4.5 \mathrm{k}$ tonnes) (FAO, 2019). By comparison, Solomon Islands produced $2.7 \mathrm{k}$ tonnes of cereals domestically in 2016 (FAO, 2019). These changes are contributing to the rising rates of obesity and non-communicable diseases (NCDs) such as heart disease and diabetes, which are now the country's leading causes of mortality (Ministry of Health and Medical Services, 2017).

Using country-level data sourced from the FAO's food balance sheets, Solomon Islands food supply was determined to have deficits in vitamin B2, vitamin A, calcium, iron, and zinc (Gibson \& Cavalli-Sforza, 2012). However, little recent dietary assessment data exist to confirm this. Most recent qualitative diet recalls show that diet diversity less than desirable, with consumption of nutritious food groups of fruits, vegetables and animal sourced foods low (Horsey et al., 2019). Government statistics indicate most Solomon Islanders currently consume fewer than five servings of fruit and vegetables per day (93.1\%), and most adults are either overweight or obese $(52.2 \%)$ (Solomon Islands National Statistics Office, 2015), contributing to the NCD epidemic of the Pacific Islands (The Lancet., 2019). Drivers of overweight and obesity are multifaceted and complex and include cultural, economic, political and individual factors (Friel et al., 2007). In the Pacific, this trend has been linked to lack of physical activity, potential genetic predisposition (especially for Indigenous populations), globalization, and the associated dietary changes that results from increased availability and 
consumption of sugar and processed imported foods (Lin et al., 2018). Largely, recommendations in the Pacific centre on reducing the consumption of foods high in sugar, salt and fat. Yet, Solomon Islands is extremely rich in local agrobiodiversity and is recognised as a "Centre of Plant Diversity". It is home to over 4500 different species of plants - 3200 of which are Indigenous and at least 120 are edible and nutrient-rich (Convention on Biological Diversity, FAO, The World Bank, UNEP, , and UNDP, 2015). No studies in the Pacific have studied the relationship between consumption patterns of local agrobiodiversity, diet quality and healthy body weight or composition outcomes.

The prevalence of undernourishment in Solomon Islands was steadily improving from $15 \%$ in 2001 to $10.6 \%$ in 2011; however, as of 2017, the percentage of undernourishment has risen to $12.3 \%$ (FAO-Stat, 2018). The percentage of children under five who are stunted has declined from 32.8\% in 2007 to $31.6 \%$ in 2015 , however wasting has increased during the same time period from $4.3 \%$ to $7.9 \%$, and childhood obesity increased from $2.5 \%$ to $3.9 \%$ (FAO-Stat, 2018). Anaemia among women of reproductive age was decreasing for a decade, but is now on the rise again, with $38.9 \%$ of women suffering from anaemia in 2016 (FAO-Stat, 2018).

Current literature investigating food system sustainability and nutrient intakes of Indigenous Solomon Islanders is limited. The population of Solomon Islands is projected to double in the next three decades, adding urgency to ensuring Indigenous food systems are resilient and able to provide sustainable diets, and food and nutrition security (UNDESA, 2012). Temperatures have increased between 0.12 and $0.18{ }^{\circ} \mathrm{C}$ each decade since the 1950 's (Solomon Islands MET, 2011). Temperature increases are a direct threat to agricultural livelihoods, including main export crops of copra and palm oil. Rising sea levels pose major threats to Indigenous Solomon Islanders, particularly coastal villages. Warming seas are changing the migratory patterns of fish, and negatively affecting both local consumption and the regional fishing industries (Pacific Adaptation Strategy Assistance Program, 2012). Pests, king tides, and flooding are additional climate-related threats that are threatening local food systems (Warrick et al., 2017). More studies are needed to examine how agrobiodiversity and dietary diversity link to improved nutrition, and can help prevent malnutrition in all its forms while promoting sustainable food systems for the future.

Within the context of a transitioning rural and Indigenous food system, this mixed-method study primarily aimed to assess the relationship between dietary agrobiodiversity and health and nutrition indicators related to diet quality and anthropometric measures. Secondary aims of this study were to evaluate the contribution of agrobiodiversity from the local food system to diet quality. The women primarily responsible for cooking of selected households were measured for height, weight and body fat and administered quantitative nutrition surveys to assess nutrient intakes over non-consecutive days, annual household food security levels, and knowledge, attitudes, and practices (KAP) around food and nutrition. The study's third objective was to capture the historical narrative around the Indigenous food system, as well as current and projected future challenges towards the promotion of sustainable food systems using participatory focus group discussions (FGDs). We aimed to use the results of these combined methodologies to identify culturally appropriate and nutritiondriven solutions towards linking agrobiodiversity, nutrition and health for more food secure and sustainable diets.

\section{Research context and methods}

\subsection{Research site}

This study took place in Baniata, a rural coastal village surrounded by forested mountains located on Rendova Island, Western Province. Baniata is home to over 645 villagers and $\sim 80$ households (Fig. 1). The soils consist of black basalt, are well drained and slightly acidic. The area receives more rain on average than anywhere else in Solomon Islands (4000 $\mathrm{mm}$ per annum), and historically has distinctive rainy and dry seasons.

Baniata is located $90 \mathrm{~min}$ by motorized boat from the nearest town of Munda, where most market exchanges occur. Villagers live largely off the land, with limited outside contact (i.e. limited cell phone and internet coverage). Touo is the local language spoken, but the research was conducted in Pidgin (common language), as Solomon Islands are home to over 75 distinct languages. While data collected only applies to one village, the vast majority of Solomon Islanders reside in rural villages with access to the sea and bush, similarly to Baniata.

\subsection{Study design}

An observational cross-sectional study design was implemented using a mixed-method approach. Quantitative surveys examined detailed dietary intake, KAP, and anthropometric measurements of woman primarily responsible for household food preparation and gardening. These quantitative data complimented seven days of participatory FGDs with a diverse representation of men and women villagers, aimed at building a narrative around perceived food system changes, as well as documenting locally available agrobiodiversity.

Data were collected from July to August 2018, which is the lean season for villagers of Baniata. Local nutritionists from Solomon Islands National University attended a multi-day training to learn the quantitative and qualitative research methodologies. Nutritionists collected data under the guidance of 
Fig. 1 Map of Baniata Village in Solomon Islands (Melanesia)*

Map was created using ArcGIS® software by Esri

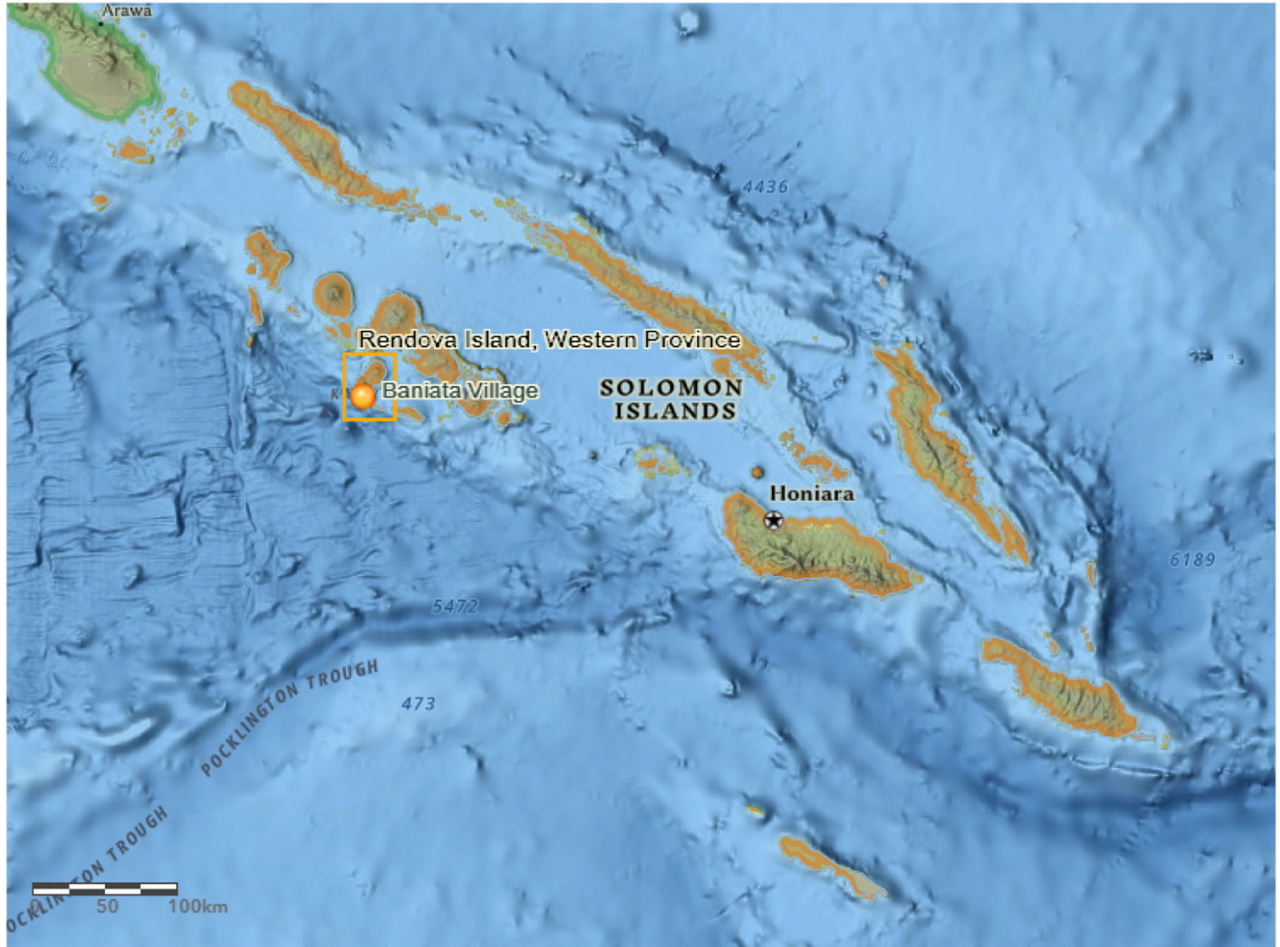

an international multi-disciplinary research team. Ethical approval was obtained from the Massey University Human Ethics Committee (\#4000019609) and research clearance from Solomon Islands Ministry of Education and Human Resources and Development (MEHRD) prior to commencing this study.

\subsection{Data collection tools}

\subsubsection{Quantitative nutrition surveys}

Nutritionist-administered surveys were used to collect quantitative data from participants using a snowball sampling technique. Inclusion criteria for the nutrition surveys were women aged 15-49 who were primarily responsible for growing, gathering, and preparing food for the household. Participants were excluded if they were pregnant or lactating, as their nutrient and energy needs are different from other adults (Butte \& King, 2005). Households were randomly selected by first generating a list of all eligible households in the village, and then using the randomization function in excel. If households were unable or unwilling to participate, a new household was randomly selected. In total, 30 women were surveyed, representing over one-third of village households $(38 \%)$. Data collection took place within the village of Baniata during the lean season in July and August 2018. Nutritionists walked to each selected household and conducted the survey within the home. The nutrition survey consisted of three sections: Anthropometry, Multiple pass quantitative 24-h dietary intake recalls, household food insecurity experience surveys (FIES), and nutrition related knowledge, attitudes and practices (KAP). The quantitative 24-h recall was repeated on a nonconsecutive day. Anthropometrics were also measured, and included height, weight, and body fat percentage.

\subsubsection{Anthropometry}

Anthropometric measurements and socio-demographic data were collected prior to beginning the nutrition survey. Height $(0.1 \mathrm{~cm})$ was assessed using a tape measure, and each nutritionist was trained accordingly with best practices (CDC, 2011). Weight and body fat percentage $(0.1 \mathrm{~kg} ; 0.1 \%)$ were assessed using a bioelectric impedance digital weight scale (GreaterGoods ${ }^{\text {TM }}$ Digital Body Fat Scale, Model 0391). BMI was calculated from the participants' height and weight $(\mathrm{BMI}=\mathrm{kg} / \mathrm{m} 2)$.

\subsubsection{4-h multiple pass recalls}

Following the anthropometric measurements, nutritionists conducted repeat, 24-h multiple-pass recalls (24 h MPR) over two non-consecutive days to capture realistic dietary intakes of the women (Gibson et al., 2017) and to capture food biodiversity (species and varietal level information of foods consumed). In addition to the quantity of food consumed, the method of cooking, species and variety, and/or brand of foods were recorded. To conduct the $24 \mathrm{~h}$ MPR, participants were prompted to provide the actual food and drinks consumed the 
previous day, from waking up till retiring. If foods consumed were a mixed dish, nutritionists had participants identify each ingredient from the recipes and recorded the amount of each ingredient used to prepare the dish, as well as the total amount (volume) available after cooking. Actual food or drinks were available $\sim 30 \%$ of the time for direct measurement. Portion sizes of actual foods and drinks were estimated by the participant and directly weighed using digital kitchen scales (Etekcity ${ }^{\mathrm{TM}}$ model EK6015) or measured using graduated cylinders $(1000 \mathrm{~mL})$. If the food or drink were not available for direct measurement, then participants estimated the portion sizes, quantity of ingredients used, and total cooked food amounts consumed using water, modelling clay or strips of paper in the original dishware. If clay or paper were used to determine portion sizes, nutritionists recorded volume displacement using graduated cylinders. After, food portions were estimated using density conversion factor estimates from the FAO/INFOODS Density Database (Charrondiere et al., 2012). Supplements and alcohol were probed for during the interview.

Participants were asked to identify the source of each ingredient; homegrown, purchased, or wild collected. Homegrown foods included foods that were intentionally managed by the household for consumption. Market purchased foods included any foods that were purchased from markets (formal or informal) or shops. Wild foods included any foods, which were not intentionally planted for consumption, and sourced from the wild including forests and the ocean.

\subsubsection{Food insecurity}

As food insecurity is often a driver of malnutrition, nutritionists administered the household Food Insecurity Experience Scale (FIES) (FAO, 2017). This eight-question item scale is designed to determine the level of household food insecurity experienced throughout the year for each household. These data were used in tandem with the qualitative food security data to triangulate the causes, timing and severity of food insecurity experienced by the villagers. The FIES is an indicator for SDG 2 (2.1.2).

\subsubsection{Knowledge, attitudes and practices}

Following FAO guidelines, a series of questions were asked assessing the participants' knowledge, attitudes, and practices (KAP) regarding nutrition (FAO, 2014) and adapted to also include food waste. The KAP questions were designed to identify specific barriers to accessing and preparing healthy foods. Data were analysed to identify how women's knowledge and attitudes influenced practices related to household food preparation. Post-harvest garden and household food waste was estimated by the primary cook of household, with primary foods and reasons for loss recorded.

\subsubsection{Focus group discussions}

A participatory rapid rural appraisal approach was designed to rapidly characterize and assess the resilience of Indigenous food systems through a series of structured discussions, using a guidebook prepared by an expert task force (SI 1). FGDs took place over the course of seven days and included participants of diverse age ranges and gender representation. Participatory FGDs were designed through a collaboration between Bioversity International and The Food and Agriculture Organization of the United Nations (UNFAO) as a part of the Indigenous Food Systems Initiative, $A$ Participatory Approach to Characterize and Access Resilience of Indigenous Food Systems to Strengthen Local Capacities and Inform Global Debates on Sustainability. Topics included traditions and trends in the food system, sustainable natural resource use, exchange trade and marketing, climatic changes, food system governance, dietary diversity, food security, and young people's knowledge and perceptions. These topics aimed to summarize the inputs and outputs of the local food system and the influencing factors in terms of food production and distribution, human health and wellbeing, and environmental impacts. Through interactive discussions, the men and women FGDs independently constructed a timeline of food system transitions over the past century. Thematic analysis used the topology of food systems presented by Chase \& Grubinger, 2014, while giving particular attention to the role of biocultural interactions and traditional knowledge.

A total of 14 participatory FGDs were held across a period of seven days and included a diverse representation of community members $(n=86)$, allowing for multigenerational insights into the challenges, strengths, and future projections regarding the sustainability of Baniata's Indigenous food system. Baniata community members - elders, women, men, and children - all have unique perspectives of the local food system with varying knowledge and responsibilities. Solomon Islands is a patriarchal society, therefore gender-specific focus groups ensured both men and women could comfortably respond to discussion questions. Open invitations were presented to all villagers for participation. Participants were organised into four focus groups based on age and gender to respect cultural gender differences and account for age-specific perspectives: men (aged 18-60; $n=26$ ), women (aged 18-60; $n=27$ ), two groups of mix-gendered children (aged 8-12 and $13-18 ; n=16$ and $n=17$ ) respectively. Male and female adult focus groups hosted $\sim 10$ people each per session, with some attending multiple days. The two groups of children participated in an abridged one-day discussion. Focus groups were facilitated by the research team in Pidgin with the 
support of local scribes, fluent in the local languages, Pidgin and English, who were tasked with taking notes and translation. Point of redundancy was considered reached for each session when either a point of consensus was reached amongst the group and/or when no further contributions were made by participants. Each session was recorded with a microphone and reanalysed to ensure an accurate translation. Themes were extracted using a peer-reviewed structural format created by Bioversity International and the UN FAO.

\subsubsection{Agrobiodiversity taxonomical identification}

Species, breed and varietal identification was completed through a local team, including an agricultural specialist, expertise from Kastom Gardens Association (local conservation organisation) and the Baniata Village Elder. Species were identified to the varietal, cultivar or breed level where possible. When not possible, species were level identification was accepted. Taxonomy was verified using The Plant List ${ }^{1}$ and The Catalogue of Life databases. ${ }^{2}$

\subsection{Data analysis}

\subsubsection{Quantitative data analysis}

Nutrition surveys, dietary data, and anthropometric measurements were analysed using IBM SPSS (Version 25), Tableau Public (Version 2018.2.2), Stata (Version 14.2), and Xyris FoodWorks (Version 9.0.3973). Nutritional composition data and food groups were sourced from the Pacific Island Food Composition Database (Version 2) (Dignan et al., 2004) and the FAO/INFOODS databases (FAO, 2018). Local foods which could not be identified in nutrition composition databases were substituted for comparable alternatives for the purpose of allocating a micronutrient profile for analysis. Usual were calculated using Multiple Source Method (MSM) (Harttig et al., 2011). Mean usual micronutrient intakes were compared with the estimated average requirements (EAR) (WHO and FAO, 2004). Usual intakes in this context are only valid for the lean season in which the data was collected, not for the entire year. The EAR estimates the average daily nutrient intake needed for half of the healthy population in a particular age and gender group. The EAR cut-point method was used to assess the proportion of participants whose usual nutrient intake falls below the EAR (National Academies Press, 2000).

Diet consumption data were categorized by the food groups outlined in the Pacific Island Food Composition Tables (v2) (Dignan et al., 2004). Ultra-processed foods were classified

\footnotetext{
$\overline{1}$ http://www.theplantlist.org/

2 http://www.catalogueoflife.org/
}

using the definitions outlined by the NOVA (1-4) method (Monteiro et al., 2019).

Four diet quality indicators were used to analyse dietary data.

1. Women's Dietary Diversity Score (WDDS) was used to determine the number of food groups women of reproductive age consumed from ten food groups, while the Minimum Dietary Diversity Score for Women (MDDW) identified the proportion of women (aged 15-49) who consumed at least $15 \mathrm{~g}$ of food from at least five of the ten food groups (FAO, 2016). Diets that contain five or more food groups have a higher propensity for achieving micronutrient adequacy (FAO, 2016). The MDD-W was designed for use with women aged 15 to 49 years of age and serves as a proxy for the probability of micronutrient adequacy. The indicator was determined from the data collected during the 24-h dietary recalls and serves as a binary indicator of adequate dietary diversity.

2. Food Variety Scores (FVS) is a validated quantitative indicator for food intake, which can be used for crosscountry data comparisons (Hodgson et al., 1994). FVS were calculated by counting the number of unique food items consumed.

3. Dietary species richness (DSR) (Lachat et al., 2018) is a count of the number of unique species that were consumed in the diet. DSR was extracted from the $24 \mathrm{~h}$ MPR data. This metric assesses both nutritional adequacy and food biodiversity, and has been validated for diets of women and children in rural areas during both wet and dry seasons.

4. Mean adequacy ratio (MAR) was calculated by summing the nutrient adequacy ratio (NAR) from ten nutrients (truncated at 100\%), and then dividing by ten.

To demonstrate the relationship between the different diet quality indicators (DSR, DDS, FVS and MDD-W) and nutrition status, a linear regression analysis was run with these variables and body fat percentage, with $p \leq 0.05$ regarded as significant. These results were also run controlling for adjusted energy intake.

Quantitative food insecurity experience scale (FIES) measures include two categories: moderate and severe. Raw scores (out of eight) between 4 and 6 were classified as moderate, and those between 6 and 8 were classified as severe (Smith et al., 2017).

\subsubsection{Estimation of misreporting of dietary intake data}

The proportion of possible over and under reporting was calculated comparing the participant's energy intake (EI) with their estimated basal metabolic rate $\left(\mathrm{BMR}_{\mathrm{est}}\right)$ using the Harris-Benedict equations (Roza \& Shizgal, 1984). The 
equations used for women is $(10 \mathrm{x}$ weight in $\mathrm{kg})+(6.25 \mathrm{x}$ height in $\mathrm{cm})-(5 \mathrm{x}$ age in years) $-161 \mathrm{x}$ PAL (physical activity level). Goldberg cut-off points were applied using the ratio between EI and $\mathrm{BMR}_{\text {est }}$. Under reporting was defined as EI: $\mathrm{BMR}_{\mathrm{est}}<1.15$ and over reporting was defined as EI: BMR $_{\text {est }}>1.96$ (Black, 2000).

\subsubsection{Qualitative data analysis}

Thematic analyses were structured from translated data into coding and classifications using the qualitative software NVIVO 12 (Version 12.1.1). Qualitative and quantitative food security data were analysed through across method triangulation (Bekhet \& Zauszniewski, 2012) combining mixed methods data to enhance the analysis and interpretation of findings.

\section{Results}

\subsection{Quantitative nutrition surveys}

\subsubsection{Participant characteristics}

Mean age of the participants was 37.1 year (range: $18-50, n=$ 30 ), and the average household size was 6.46 , typically consisting of a multi-generational family. Agri-food sales were the primary source of income for all participants and provided an average monthly household income between \$500-1500 SBD (\$63-188 USD). The majority of women $(60 \%)$ were classified as overweight or obese, with a mean BMI of 26 (SD 4.9). Women had a median body fat percentage of $31.1 \%$ (SD 7.6) (Table 1).

\subsubsection{Dietary intake}

Mean MAR was found to be $0.70(\mathrm{SD} \pm .16)$. Average macronutrient distributions indicated energy from fat (40\%) was above the acceptable macronutrient distribution range for adults of 20-35\%; energy from protein was low (9\%), below the acceptable range of 10-35\%; whilst energy from carbohydrate $(51 \%)$ fell within the acceptable range of $45-65 \%$ of total energy intake.

Our data indicate that usual intakes of nutrients during the end of the lean season were significantly below the EAR (Table 2). Women who have inadequate intakes of essential nutrients include protein (53\%), calcium $(96.6 \%)$, vitamin $\mathrm{B}_{1}$ $(87 \%)$, vitamin $\mathrm{B}_{2}(80 \%)$, vitamin $\mathrm{A}(80 \%)$, and fibre (76.7\%). A half $(52.3 \%)$ of women had a sodium intake above the WHO (2012) guidelines of $>2 \mathrm{~g} /$ day. Energy intake was also slightly below average, with $63 \%$ of women consuming fewer calories than recommended. Estimation of misreporting
Table 1 Household and participant sociodemographic characteristics $(\mathrm{n}=30)$

\begin{tabular}{|c|c|}
\hline & $n$ \\
\hline \multicolumn{2}{|l|}{ Household characteristics $(n=30)$} \\
\hline \multicolumn{2}{|l|}{ Annual incomes } \\
\hline$<\$ 12,000 S B D(<\$ 1470$ USD $)$ & 18 \\
\hline$>\$ 12,000 S B D(>\$ 1470$ USD $)$ & 12 \\
\hline \multicolumn{2}{|l|}{ Household size } \\
\hline $\operatorname{Low}(1-4)$ & 7 \\
\hline Medium (5-9) & 18 \\
\hline $\operatorname{High}(10-14)$ & 5 \\
\hline \multicolumn{2}{|l|}{ Religion observed } \\
\hline Seventh Day Adventist & 12 \\
\hline Christian Fellowship Church (CFC) & 18 \\
\hline \multicolumn{2}{|l|}{ Participant characteristics ( $\mathrm{n}=30$ women) } \\
\hline \multicolumn{2}{|l|}{ Highest education received } \\
\hline Primary school & 25 \\
\hline Secondary school or higher & 5 \\
\hline \multicolumn{2}{|l|}{ Drug usage (daily) } \\
\hline Cigarettes & 9 \\
\hline Betel nut & 21 \\
\hline Alcohol & 0 \\
\hline \multicolumn{2}{|l|}{ Ages of participant (year) } \\
\hline Mean & 37.1 \\
\hline Min & 18 \\
\hline $\operatorname{Max}$ & 50 \\
\hline \multicolumn{2}{|l|}{ Body Fat Percentage $(\%)$} \\
\hline Mean & 30.1 \\
\hline Min & 17.2 \\
\hline $\operatorname{Max}$ & 43.1 \\
\hline \multicolumn{2}{|l|}{ Body Mass Index (BMI) } \\
\hline Mean & 26 \\
\hline Min & 16.7 \\
\hline $\operatorname{Max}$ & 35.1 \\
\hline
\end{tabular}

at the group level via the Goldberg cut off points (1.44) indicated that no significant under- or overreporting occurred.

The macro and micronutrient contribution of homegrown, wild collected, and purchased (primarily imported) foods varied considerably per nutrient (Table 3 ). Most energy consumed came from homegrown foods $(50.8 \%)$ followed by purchased foods (35.3\%) and finally wild foods (13.9\%). Commonly consumed homegrown foods included root vegetables, coconuts, bananas, cabbages, breadfruit and nuts. Only $13.3 \%$ of women met the WHO/FAO joint recommendation (FAO and WHO, 2004) to consume more than $400 \mathrm{~g}$ of fruits and vegetables per day (excluding starchy tubers and potatoes). Purchased foods constituted over a third (34.1\%) of total energy intake, with ultra-processed (NOVA 4) foods contributing $11.3 \%$ of energy intakes. Top energy contributions from 
Table 2 Usual nutrient intake of women in Baniata Village, Solomon Islands, calculated from 24-h multiple pass dietary recalls and compared to recommended intakes $(n=30)$

\begin{tabular}{|c|c|c|c|c|}
\hline & Mean & SD & $\%$ of women below $\mathrm{EAR}_{\dagger}$ & $\%$ women above recs \\
\hline \multicolumn{5}{|l|}{ Macronutrients } \\
\hline Energy $(\mathrm{kJ}) \ddagger$ & 7648 & 2540 & $63.3 \%$ & \\
\hline Energy (Kcal) & 1828 & 607 & $63.3 \%$ & \\
\hline Total fat $(\mathrm{g}) *$ & 79.8 & 35.4 & - & $76.7 \%$ \\
\hline$\%$ total diet & $40.3 \%$ & & & \\
\hline Saturated fat $(\mathrm{g})^{*}$ & 52.6 & 29.1 & - & $70 \%$ \\
\hline Protein $(g)$ & 40.7 & 17.2 & $53.3 \%$ & \\
\hline$\%$ total diet & $9.2 \%$ & & & \\
\hline Carbohydrates (g) & 225 & 79.1 & $6.7 \%$ & \\
\hline$\%$ total diet & $50.5 \%$ & & & \\
\hline Sugar $(g)$ & 61.1 & 20.7 & - & $66.6 \%$ \\
\hline Fibre $(\mathrm{g})$ & 20.1 & 9.61 & $76.6 \%$ & \\
\hline \multicolumn{5}{|l|}{ Micronutrients } \\
\hline Vitamin A $(\mu \mathrm{g})$ & 347.8 & 394 & $80 \%$ & \\
\hline Vitamin $\mathrm{B}_{1}(\mathrm{mg})$ & 0.63 & 0.37 & $86.6 \%$ & \\
\hline Vitamin $B_{2}(\mathrm{mg})$ & 0.61 & 0.36 & $80 \%$ & \\
\hline Vitamin $B_{3}(\mathrm{mg})$ & 20.5 & 8.42 & $13.3 \%$ & \\
\hline Vitamin C (mg) & 84.7 & 56.7 & $13.3 \%$ & \\
\hline $\mathrm{Na}(\mathrm{mg}) *$ & 1934 & 842 & - & $53.3 \%$ \\
\hline $\mathrm{K}(\mathrm{mg})$ & 3204 & 1548 & $50 \%$ & \\
\hline $\mathrm{Ca}(\mathrm{mg})$ & 290.7 & 233 & $96.6 \%$ & \\
\hline $\mathrm{Mg}(\mathrm{mg})$ & 417 & 261 & $33 \%$ & \\
\hline P (mg) & 613 & 252 & $50 \%$ & \\
\hline $\mathrm{Fe}(\mathrm{mg})$ & 11.4 & 6.6 & $30 \%$ & \\
\hline $\mathrm{Zn}(\mathrm{mg})$ & 8.18 & 2.93 & $40 \%$ & \\
\hline
\end{tabular}

$\uparrow$ Percentage of women below the estimated average nutrient requirements (EAR) for adults. Total fat, saturated fat, sugar, and sodium do not have an EAR for minimum quantity

$\ddagger$ Energy requirements were compared to total energy expenditure (TEE) from BMR + PAL

*Total fat recommendations $<30 \%$ total kcal and saturated fat recommendations $<10 \%$ of total kcal (Hooper et al., 2015), Na recommendations <2000 mg (WHO, 2012)
NOVA 1-3 categories are white rice, tayio (canned tuna), and table sugar. Energy from NOVA 4 ultra-processed foods are sourced from noodles, biscuits, sugar sweetened beverages (Milo), and baked sweets. Whilst wild foods were a small contributor to the diet, they were micronutrient dense foods most commonly including ferns, opossums, fish, crabs, and shellfish.

Table 4 features the energy and macronutrient consumption percentages for each food group, as classified by the Pacific Islands food composition tables. Over $60 \%$ of mean daily energy intake came from just 3 main food types, primarily coconut products (that including flesh, milk/cream, and oil $-23.3 \%$ ), roots and tubers $(21.8 \%)$ and cereals and grains (mainly rice $16.3 \%$ ). Fish (mostly tinned) and bananas (desert and cooking) together made up just over $15 \%$ of total energy intake. Protein consumption was low (40.71 $\mathrm{g} \pm \mathrm{SD}$ 17.27), fish provided most of daily protein $(32.3 \%)$, followed by cereals and grains (19.2\%). Half of daily fat intakes came from coconut products, followed by fish (10.7\%) and store- bought refined oils (11.1\%).
Out of a score of 10, MDD-W ranged from two to six (average $4.18 \mathrm{SD} \pm 0.96)$ and FVS averaged $7.5( \pm \mathrm{SD}$ 2.3). Over two-thirds (73\%) of participants did not meet MDD-W, consuming fewer than five food groups. Starchy foods (roots, tubers and bananas) were the most commonly consumed food groups (97\%) and were present in nearly every meal. Fish (90\%), followed by dark leafy green vegetables $(83 \%)$ were also commonly consumed, whereas pulses, eggs, and dairy were the food groups least likely to be consumed $(17 \%, 7 \%$, and $0 \%$, respectively). There was a difference between the food groups consumed by those who reached MDD-W and those that did not (Table 5). In particular, from those that reached MDDW, fewer consumed grains $(-25 \%)$ and replaced it with higher prevalence of consumption of nutrient rich foods, in particular nuts and seeds $(+47 \%$ - mainly ngali nut), pulses $(+28 \%)$, Vitamin A rich fruits $(+26 \%)$, DGLV $(+23 \%)$, fish and seafood $(+14 \%)$, and meat and eggs (+8\% for each). 
Table 3 Macronutrient and micronutrient contributions from homegrown, wild collected, and purchased foods in Baniata

Village, Solomon Islands $(\mathrm{n}=30$ women)

\begin{tabular}{|c|c|c|c|c|}
\hline & Homegrown & Wild collected & $\begin{array}{l}\text { Purchased } \\
\text { NOVA } 1-3 *\end{array}$ & $\begin{array}{l}\text { Purchased } \\
\text { NOVA } 4 *\end{array}$ \\
\hline \multicolumn{5}{|l|}{ Macronutrients } \\
\hline Energy & $51.7 \%$ & $14.2 \%$ & $22.8 \%$ & $11.3 \%$ \\
\hline Protein & $34.9 \%$ & $33.8 \%$ & $22.3 \%$ & $8.9 \%$ \\
\hline Total fat & $59.0 \%$ & $23.2 \%$ & $5.2 \%$ & $12.6 \%$ \\
\hline Saturated fat & $64.3 \%$ & $21.4 \%$ & $3.5 \%$ & $10.8 \%$ \\
\hline Carbohydrates & $48.5 \%$ & $4.9 \%$ & $35.3 \%$ & $11.3 \%$ \\
\hline Fibre & $51.6 \%$ & $9.3 \%$ & $29.3 \%$ & $9.8 \%$ \\
\hline Micronutrients & $73.4 \%$ & $13.6 \%$ & $8.4 \%$ & $4.6 \%$ \\
\hline Vitamin A & $87.8 \%$ & $11.4 \%$ & $0.1 \%$ & $0.7 \%$ \\
\hline Vitamin $B_{1}$ & $60.5 \%$ & $12.9 \%$ & $8.5 \%$ & $18.1 \%$ \\
\hline Vitamin $B_{2}$ & $65.3 \%$ & $16.6 \%$ & $12.0 \%$ & $6.2 \%$ \\
\hline Vitamin $B_{3}$ & $45.7 \%$ & $19.5 \%$ & $26.3 \%$ & $8.5 \%$ \\
\hline Vitamin C & $90.2 \%$ & $9.1 \%$ & $0.2 \%$ & $0.5 \%$ \\
\hline $\mathrm{Na}$ & $17.9 \%$ & $13.4 \%$ & $57.3 \%$ & $11.3 \%$ \\
\hline K & $78.9 \%$ & $15.6 \%$ & $2.8 \%$ & $2.7 \%$ \\
\hline $\mathrm{Ca}$ & $79.0 \%$ & $13.3 \%$ & $4.4 \%$ & $3.4 \%$ \\
\hline $\mathrm{Mg}$ & $68.4 \%$ & $21.9 \%$ & $7.5 \%$ & $2.2 \%$ \\
\hline $\mathrm{Fe}$ & $62.1 \%$ & $17.9 \%$ & $6.0 \%$ & $14.0 \%$ \\
\hline $\mathrm{Zn}$ & $38.9 \%$ & $16.2 \%$ & $36.9 \%$ & $8.0 \%$ \\
\hline Iodine & $17.2 \%$ & $14.2 \%$ & $65.2 \%$ & $3.4 \%$ \\
\hline
\end{tabular}

*NOVA classifications: (1) unprocessed and minimally processed foods (2) processed culinary ingredients (3) processed foods (4) ultra-processed foods

Table 4 Energy and

macronutrient consumption percentages of women in Baniata Village, Solomon Islands, grouped by Pacific Island food groups* $(n=30)$

\begin{tabular}{lllll}
\hline Food group & $\%$ Total energy $(\mathrm{kJ})$ & $\%$ Total protein & $\%$ Total fat & $\begin{array}{l}\% \text { Total } \\
\text { carbohydrates }\end{array}$ \\
\hline Coconut products & $23.3 \%$ & $11.8 \%$ & $50.3 \%$ & $3.5 \%$ \\
Starchy veg (roots, tubers) & $21.8 \%$ & $11.7 \%$ & $3.6 \%$ & $35.9 \%$ \\
Cereals and grain products (rice) & $16.3 \%$ & $19.2 \%$ & $1.3 \%$ & $26.7 \%$ \\
Processed foods & $10.5 \%$ & $7.9 \%$ & $11.1 \%$ & $10.3 \%$ \\
Fish & $7.4 \%$ & $32.4 \%$ & $10.7 \%$ & $0.3 \%$ \\
Bananas & $7.2 \%$ & $3.4 \%$ & $0.5 \%$ & $12.3 \%$ \\
Fruits & $4.3 \%$ & $2.4 \%$ & $7.7 \%$ & $1.9 \%$ \\
Confectionary (inc. sugar) & $4.2 \%$ & $0.0 \%$ & $0.0 \%$ & $8.1 \%$ \\
Nuts and seeds & $3.1 \%$ & $5.1 \%$ & $11.8 \%$ & $0.4 \%$ \\
Seafood (non-fish) & $0.5 \%$ & $3.0 \%$ & $0.5 \%$ & $0.1 \%$ \\
Green vegetables & $0.4 \%$ & $0.8 \%$ & $0.7 \%$ & $0.3 \%$ \\
Fats and oils (exc. coconut) & $0.4 \%$ & $0.0 \%$ & $0.9 \%$ & $0.0 \%$ \\
Eggs & $0.3 \%$ & $1.3 \%$ & $0.5 \%$ & $0.0 \%$ \\
Vegetables & $0.2 \%$ & $0.3 \%$ & $0.1 \%$ & $0.2 \%$ \\
Legumes & $0.1 \%$ & $0.5 \%$ & $0.0 \%$ & $0.1 \%$ \\
Herbs, spices, sauces & $0.1 \%$ & $0.1 \%$ & $0.1 \%$ & $0.0 \%$ \\
Meat and poultry & $0.1 \%$ & $0.2 \%$ & $0.1 \%$ & $0.0 \%$ \\
Beverages & $0.0 \%$ & $0.0 \%$ & $0.0 \%$ & $0.0 \%$ \\
\hline
\end{tabular}

*Food groups were categorized in accordance with the Pacific Island food composition tables 
Table 5 Food group consumption patterns split by those who achieved minimum diet diversity scores of $\geq 5$ compared with those who had scores $<5(\mathrm{n}=30)$

\begin{tabular}{lll}
\hline & DDS $<5(\mathrm{n}=22)$ & $\mathrm{DDS} \geq 5(\mathrm{n}=8)$ \\
\hline Foods from grains & $100.00 \%$ & $75.00 \%$ \\
White roots, plantains, tubers & $95.45 \%$ & $100.00 \%$ \\
Pulses (beans, peas, lentils) & $9.09 \%$ & $37.50 \%$ \\
Nuts / seeds & $40.91 \%$ & $87.50 \%$ \\
Milk and milk products & $0.00 \%$ & $0.00 \%$ \\
Organ meat & $0.00 \%$ & $0.00 \%$ \\
Meat and poultry & $4.55 \%$ & $12.50 \%$ \\
Fish and seafood & $86.36 \%$ & $100.00 \%$ \\
Eggs & $4.55 \%$ & $12.50 \%$ \\
Dark green leafy vegetables & $77.27 \%$ & $100.00 \%$ \\
Vit a rich roots/tubers & $22.73 \%$ & $25.00 \%$ \\
Vit a rich fruits & $9.09 \%$ & $25.00 \%$ \\
Other vegetables & $22.73 \%$ & $12.50 \%$ \\
Other fruits & $81.82 \%$ & $87.50 \%$ \\
\hline
\end{tabular}

The agrobiodiversity of foods reported to be consumed by participants included 39 different species and, in total, 99 different varieties and breeds (Table 6). This does not include purchased processed foods with more than one ingredient. Food groups with the greatest species richness found to be consumed were roots, tubers and bananas (33), seafood (23), dark green leafy vegetables (11), fruits (11), non-leafy vegetables (7), nuts and seeds (4), legumes (3), eggs (2), animals (2), and 1 grain (white rice). Average DSR was 12.1 (SD 3.4). DSR was statistically significantly positively correlated with MAR (Coef. 0.039, $p<0.05$,).

The strength of the inverse associations between DSR, FVS, DDS and body fat percentage were weak $(-0.205,0.231$ and 0.251 respectively) but statistically significant ( $p=0.012$, 0.004 , and 0.007 respectively) (Fig. 2). These associations were still evident and significant after controlling for energy intake (SI 2). MAR was not significantly related to body fat percentage (Coef-4.93, $p=0.402$ ). Reported levels of income did not show significant association with body fat percentage. More food secure households were associated with higher body fat percentages, though not significant. Knowledge attitudes and practices towards the importance of fruits and vegetable consumption were generally low (Table 7).

\subsubsection{Household food insecurity experience scale}

Household food insecurity was seasonally variable, with villagers experiencing the highest self-reported rates during the lean months of May, June and July. Of the 30 households surveyed, $48 \%$ were moderately food insecure, and $10.3 \%$ were severely food insecure, primarily during the lean season.
Results from the FIES indicated that throughout the year due to lack of money or other resources: $83 \%$ of households were worried they would not have enough food; $77 \%$ ate less than they thought they should; $60 \%$ ate fewer types of foods; $57 \%$ were unable to eat healthy foods and/or were hungry and did not eat; $42 \%$ skipped a meal; $28 \%$ ran out of food; and $4 \%$ went an entire day without eating.

\subsubsection{Food wastage and preservation}

Participants estimated that $27 \%$ of all food was wasted via either post-harvest losses or household losses. Top factors for food waste included cooking too much food (87\%), over collection of foods $(67 \%)$, did not use in time $(63 \%)$, too much grown (57\%), or forgot/did not want to use $(23 \%)$. The most commonly wasted foods were roots/tubers $(90 \%)$, vegetables $(50 \%)$, fruits $(43 \%)$, and nuts/seeds (13\%). Negligible amounts of meat or fish was wasted. Food scraps were often left for free-roaming chickens and hogs to forage or discarded with trash. Very little food waste was composted or returned to the gardens for nutrient recycling.

Food preservation was limited to dried ngali nuts and smoked fish. Most households (87\%) processed and dried ngali nuts for household consumption and agri-food sales. A small number of households practised smoking fish for preservation (23\%). No other food preservation techniques were reported.

\subsection{Qualitative participatory focus group discussions}

\subsubsection{History of food system transitions}

Food system transitions away from traditional diets were reported (by men and women) to begin as early as the 1920's during visits from Seventh Day Adventist missionaries (Fig. 3). As an example, Seventh Day Adventistism prohibits the consumption of certain foods, such as pigs, possum, molluscs, and crustaceans. Dietary shifts again occurred during World War II (WWII) when American and Japanese soldiers were living amongst the villagers. One Baniata woman explained that "dietary changes occurred following WWII when the war was over and remaining rations were shared among the villagers". These rations consisted of packaged noodles, canned meats, and sugar. During the 1970's, it was reported that the Solomon Islands government provided subsidies for villagers to increase production of coconuts for agri-food income generation opportunities. In the late 1970s, Solomon Tayio limited, a large tuna processing facility, opened for production in Noro town, located around two hours from the village by boat. Once established, diets were said to begin transitioning from fresh fish to canned Solomon tayio (tuna) due to ease of access and affordability.

The most dramatic dietary transitions were reported by the elders to have occurred within the past 2-3 decades. One man 
Table 6 Agrobiodiversity data informed by focus group discussions in Baniata Village, Solomon Islands

\begin{tabular}{|c|c|c|c|c|}
\hline Crop species & Common English Name & Local name & Description & $\begin{array}{l}\text { Consumed in } \\
24 \mathrm{~h} \mathrm{MPR}\end{array}$ \\
\hline \multicolumn{5}{|l|}{ Roots and Tubers } \\
\hline Alocasia macrorrhiza (L.) Schott & Giant Taro & Ozo & White flesh & \\
\hline Alocasia macrorrhiza (L.) Schott & Giant Taro & Ozo & Dark red/pink flesh & \\
\hline Colocasia esculenta (L.) Schott & Taro & Fivo/Buini & - & \\
\hline Colocasia esculenta (L.) Schott & Taro & Mahio & - & \\
\hline Colocasia esculenta (L.) Schott & Taro & Omu & - & $\mathrm{X}$ \\
\hline Colocasia esculenta (L.) Schott & Taro & Ruta & - & \\
\hline Colocasia esculenta (L.) Schott & Taro & Sisiri & - & \\
\hline Colocasia esculenta (L.) Schott & Taro & Sofu & - & \\
\hline Cyrtosperma merkusii (Hassk.) Schott. & Swamp Taro & Kakake & - & $\mathrm{X}$ \\
\hline Dioscorea alata $L$ & Greater Yam & Purple Yam & Purple flesh & $\mathrm{X}$ \\
\hline Dioscorea alata $L$. & Greater Yam & White Yam & White flesh & $\mathrm{X}$ \\
\hline Dioscorea esculenta (Loureiro) Burkill & Pana or Lesser Yam & Finorusu & - & \\
\hline Dioscorea esculenta (Loureiro) Burkill & Pana or Lesser Yam & Fivo pana & Purple flesh & $\mathrm{X}$ \\
\hline Dioscorea esculenta (Loureiro) Burkill & Pana or Lesser Yam & Susa & - & \\
\hline Dioscorea esculenta (Loureiro) Burkill & Pana or Lesser Yam & Ulawa & - & \\
\hline Dioscorea rotundata Poir & Greater Yam & Vanuatu Long & - & \\
\hline Dioscorea rotundata Poir & Greater Yam & Vanuatu Short & - & \\
\hline Dioscorea spp. & Greater Yam & Butterfly Yam & White flesh & $\mathrm{X}$ \\
\hline Dioscorea spp. & Greater Yam & Hero Yam & Red and white flesh & \\
\hline Dioscorea spp. & Greater Yam & Hoahoa & - & \\
\hline Dioscorea spp. & Greater Yam & Riseboy & Purple flesh & \\
\hline Dioscorea spp. & Greater Yam & Snake Yam & White flesh & \\
\hline Dioscorea spp. & Greater Yam & Tonga yam & - & \\
\hline Dioscorea spp. & Greater Yam & Vaka Yam & White flesh & \\
\hline Dioscorea spp. & Greater Yam & Vero & - & $\mathrm{X}$ \\
\hline Dioscorea spp. & Pana or Lesser Yam & Bou & - & \\
\hline Ipomoea batatas (Linnaeus) Lam. & Sweet potato & Atoifi & Red skin, yellow flesh & $X$ \\
\hline Ipomoea batatas (Linnaeus) Lam. & Sweet potato & Benimala & Purple flesh & \\
\hline Ipomoea batatas (Linnaeus) Lam. & Sweet potato & Ema Duri & Yellow flesh & $X$ \\
\hline Ipomoea batatas (Linnaeus) Lam. & Sweet potato & Fivomahu & - & \\
\hline Ipomoea batatas (Linnaeus) Lam. & Sweet potato & Meleke & Purple flesh & $\mathrm{X}$ \\
\hline Ipomoea batatas (Linnaeus) Lam. & Sweet potato & Nimbi & - & \\
\hline Ipomoea batatas (Linnaeus) Lam. & Sweet potato & Taeveke & Red skin, white flesh & $\mathrm{X}$ \\
\hline Ipomoea batatas (Linnaeus) Lam. & Sweet potato & Taumahu & - & \\
\hline Ipomoea batatas (Linnaeus) Lam. & Sweet potato & Temarae & White flesh & $\mathrm{X}$ \\
\hline Ipomoea batatas (Linnaeus) Lam. & Sweet potato & Two Months & - & \\
\hline Ipomoea batatas (Linnaeus) Lam. & Sweet potato & Vaero & Light yellow flesh & \\
\hline Manihot esculenta Crantz & Cassava & Fizi & White flesh & \\
\hline Manihot esculenta Crantz & Cassava & Green Top & - & \\
\hline Manihot esculenta Crantz & Cassava & Kaiza & - & $\mathrm{X}$ \\
\hline Manihot esculenta Crantz & Cassava & Pencil Cassava & White flesh & $\mathrm{X}$ \\
\hline Manihot esculenta Crantz & Cassava & Ranoga & - & $\mathrm{X}$ \\
\hline Manihot esculenta Crantz & Cassava & Six Months & White flesh & \\
\hline Manihot esculenta Crantz & Cassava & Underpant & White flesh & $\mathrm{X}$ \\
\hline Manihot esculenta Crantz & Cassava & Yellow Curry & Yellow flesh & $X$ \\
\hline Xanthosoma sagittifolium (L.) Schott & Chinese Taro & Karuver & White flesh & $X$ \\
\hline Xanthosoma sagittifolium (L.) Schott & Chinese Taro & Karuvera & Pink flesh & $\mathrm{X}$ \\
\hline
\end{tabular}


Table 6 (continued)

\begin{tabular}{|c|c|c|c|c|}
\hline Crop species & Common English Name & Local name & Description & $\begin{array}{l}\text { Consumed in } \\
24 \mathrm{~h} \mathrm{MPR}\end{array}$ \\
\hline Zingiber officinale Roscoe & Ginger & Tuva Migori & White flesh & $X$ \\
\hline Zingiber officinale var. Rubrum Theilade & Ginger & Aro Migori & Red flesh & $\mathrm{X}$ \\
\hline \multicolumn{5}{|l|}{ Banana } \\
\hline Musa sp (A\&/or B genome) cv. & Banana & Bo’o & White flesh & \\
\hline Musa sp (A \&/or B genome) cv. & Banana & Bougainville & White/yellow & $\mathrm{X}$ \\
\hline Musa sp (A \&/or B genome) cv. & Banana & Gatokae & & $\mathrm{X}$ \\
\hline Musa sp (A \&/or B genome) cv. & Banana & Hiomo & - & \\
\hline Musa sp (A \&/or B genome) cv. & Banana & Misisi & Yellow flesh & \\
\hline Musa sp (A \&/or B genome) cv. & Banana & Napoti & Yellow flesh & $\mathrm{X}$ \\
\hline Musa sp (A \&/or B genome) cv. & Banana & Pohara & White flesh & $\mathrm{X}$ \\
\hline Musa sp (A \&/or B genome) cv. & Banana & Rabaul & - & \\
\hline Musa sp (A \&/or B genome) cv. & Banana & Richard & - & $X$ \\
\hline Musa sp (A \&/or B genome) cv. & Banana & Twistie & - & $\mathrm{X}$ \\
\hline Musa sp (A \&/or B genome) cv. & Banana & Uvi & Yellow flesh & $\mathrm{X}$ \\
\hline Musa sp (A \&/or B genome) cv. & Banana & Vasara & Yellow flesh & $\mathrm{X}$ \\
\hline Musa sp (A \&/or B genome) cv. & Banana & Wasara Vahu & & $\mathrm{X}$ \\
\hline (Musa spp) & Banana & Fiji Vahu & Yellow flesh & $X$ \\
\hline (Musa spp) & Banana & Nuguru & - & $X$ \\
\hline (Musa spp) & Banana & Pedi Vahu & - & \\
\hline (Musa spp) & Banana & Sikaiana Vahu & - & \\
\hline (Musa spp) & Banana & Sweet Banana & Yello and white flesh & $X$ \\
\hline (Musa spp) & Banana & Zario Vahu & - & \\
\hline \multicolumn{5}{|l|}{ Fruit } \\
\hline Ananas comosus (L.) Merr. & Pineapple & Pineapple & Brown skin, yellow flesh & \\
\hline Ananas comosus (L.) Merr. & Queen Pineapple & Pineapple & Brown skin, yellow flesh & \\
\hline Annona muricata $\mathrm{L}$. & Soursop & Soursop/omo & Green skin, white flesh & $\mathrm{X}$ \\
\hline Artocarpus altilis (Parkinson) Fosberg & Breadfruit & me'u & Green flesh & $\mathrm{X}$ \\
\hline Averrhoa carambola $L$ & Carambola & Starfruit & Yellow skin, pale flesh & $\mathrm{X}$ \\
\hline Bruguiera gymnorhiza (L.) Lam. & Mangrove fruit & Fetu & & $\mathrm{X}$ \\
\hline Burckella obovata (G.Forst.) Pierre & Burckella & Sohvao & $\begin{array}{l}\text { Green outer skin and yellow } \\
\text { flesh }\end{array}$ & \\
\hline Unidentified & Unidentified & Voh & $\begin{array}{l}\text { Yellow outer skin and white } \\
\text { flesh }\end{array}$ & \\
\hline Carica papaya $\mathrm{L}$. & Pawpaw/Papaya & Mango - Paw paw & Orange flesh & $\mathrm{X}$ \\
\hline Carica papaya L. & Pawpaw/Papaya & Melon - paw paw & Red flesh & \\
\hline Carica papaya L. & Pawpaw/Papaya & Paw paw & Yellow skin & $\mathrm{X}$ \\
\hline $\begin{array}{l}\text { Citrullus lanatus (Thunb.) } \\
\text { Matsum. \& Nakai }\end{array}$ & Watermelon & Melon & Yellow and red flesh, long & \\
\hline $\begin{array}{l}\text { Citrullus lanatus (Thunb.) } \\
\text { Matsum. \& Nakai }\end{array}$ & Watermelon & Melon & Red and yellow flesh, round & \\
\hline Citrus aurantium L. & Sour Orange & Half Orange & Orange flesh & \\
\hline Citrus grandis (L.) Osbeck & Pomelo & Pomolo & White flesh & \\
\hline Citrus grandis (L.) Osbeck & Pomelo & Pomolo & Pink flesh & $\mathrm{X}$ \\
\hline Citrus grandis (L.) Osbeck & Pomelo & Pomolo & - & $\mathrm{X}$ \\
\hline Citrus reticulata Blanco & Mandarin & Madarin & Orange flesh & \\
\hline Citrus sinensis (L.) Osbeck & Orange & Sweet orange & Orange flesh & \\
\hline Cocos nucifera $\mathrm{L}$. & Coconut & Coconut & Green skin & $X$ \\
\hline Magnifera indica $\mathrm{L}$. & Mango & Mango & $\begin{array}{l}\text { Light yellow or White flesh, } \\
\text { wild }\end{array}$ & \\
\hline Magnifera indica L. & Mango & Mango & Orange flesh, common & \\
\hline
\end{tabular}


Table 6 (continued)

\begin{tabular}{|c|c|c|c|c|}
\hline Crop species & Common English Name & Local name & Description & $\begin{array}{l}\text { Consumed in } \\
24 \mathrm{~h} \mathrm{MPR}\end{array}$ \\
\hline Nephelium lappaceum $\mathrm{L}$. & Rambutan & Rambutan & White flesh & \\
\hline Persia americana Mill & Avocado & Avocado & Green flesh & \\
\hline Pometia pinnata J.R.Forst. \& G.Forst. & Pacific Lychee & Gema fruit & - & \\
\hline Psidium guajava $\mathrm{L}$. & Guava & Guava & Pink flesh, local, small & $\mathrm{X}$ \\
\hline Psidium guajava L. & Guava & Guava & White flesh, big, long & $\mathrm{X}$ \\
\hline Psidium guajava L. & Guava & Guava & Pink flesh, big, round & \\
\hline Spondias cytherea Sonn & Golden apple & Encori or opiti & White and yellow flesh & \\
\hline Syzygium aqueum (Burm.f.) Alston & Watery rose apple & Kapicala & PNG & \\
\hline $\begin{array}{l}\text { Syzygium malaccense (L.) Merr. \& } \\
\text { L.M.Perry }\end{array}$ & Malay apple & Kapicala & $\mathrm{Big} / \mathrm{small}$ & \\
\hline $\begin{array}{l}\text { Syzygium malaccense (L.) Merr. \& } \\
\text { L.M.Perry }\end{array}$ & Malay apple & Kapicala & Wild & \\
\hline \multicolumn{5}{|l|}{ Beans and Legumes } \\
\hline Arachis hypogea L. & Peanut & Peanut & White, flat & \\
\hline Arachis hypogea L. & Peanut & Peanut & Red & \\
\hline Arachis hypogea L. & Peanut & Peanut & Purple & \\
\hline Arachis hypogea L. & Peanut & Peanut & White & \\
\hline Arachis hypogea L. & Peanut & Peanut & Red & $\mathrm{X}$ \\
\hline Benincasa hispida (Thunb.) Cogn. & Wax Gourd & Waku bean & Big white, round & \\
\hline Psophocarpus tetragonolobus (L.) DC. & Wing bean & Butterfly bean & - & \\
\hline Trichosanthes cucumerina $L$ & Snake gourd & (Cocoa) Snakebean & Cocoa, short and wide & $\mathrm{X}$ \\
\hline $\begin{array}{l}\text { Trichosanthes cucumerina var. anguina } \\
\text { L. }\end{array}$ & Snake gourd & Snakebean & Striped body, Army & $\mathrm{X}$ \\
\hline $\begin{array}{l}\text { Vigna unguiculata subsp. sesquipedalis } \\
\text { (L) Verdic }\end{array}$ & Purple YardLong Bean & Rigiti Bean & Purple & \\
\hline $\begin{array}{l}\text { Vigna unguiculata subsp. Sesquipedalis } \\
\text { (L) Verdic }\end{array}$ & Striped YardLong Bean & Rigiti Bean & Army & $\mathrm{X}$ \\
\hline $\begin{array}{l}\text { Vigna unguiculata subsp. Sesquipedalis } \\
\text { (L) Verdic }\end{array}$ & YardLong Bean & Rigiti bean & Black bean / seeds & \\
\hline $\begin{array}{l}\text { Vigna unguiculata subsp. Sesquipedalis } \\
\text { (L) Verdic }\end{array}$ & YardLong Bean & Rigiti bean & Small & $\mathrm{X}$ \\
\hline $\begin{array}{l}\text { Vigna unguiculata subsp. Sesquipedalis } \\
\text { (L) Verdic }\end{array}$ & YardLong Bean & Green bean & Long & \\
\hline $\begin{array}{l}\text { Vigna unguiculata subsp. Sesquipedalis } \\
\text { (L) Verdic }\end{array}$ & YardLong Bean & Waku bean & Long, large/thicker pod & \\
\hline Vigna unguiculata var. unguiculata & Cowpea or dwarf bean & Cowpea bean & dwarf & \\
\hline \multicolumn{5}{|l|}{ Dark Green Leafy Vegetables } \\
\hline Abelmoschus manihot (L.) Medic. & Hibiscus/Slippery cabbage & $\begin{array}{l}\text { Slippery cabbage - } \\
\text { noodle }\end{array}$ & & $\mathrm{X}$ \\
\hline Abelmoschus manihot (L.) Medic. & Hibiscus/Slippery cabbage & $\begin{array}{l}\text { Slippery cabbage - } \\
\text { Tsunami }\end{array}$ & & $\mathrm{X}$ \\
\hline Abelmoschus manihot (L.) Medic. & Hibiscus/Slippery cabbage & $\begin{array}{l}\text { Slippery cappage - } \\
\text { maruana }\end{array}$ & & \\
\hline Allium cepa var. aggregatum L., G.Don. & Spring onion/Bunching onion & Shallot & & $\mathrm{X}$ \\
\hline Brassica campestris $\mathrm{L}$. & Saladeer & Saladia & & \\
\hline Brassica rapa $\mathrm{L}$. & Chinese cabbage & Paksoi & & $\mathrm{X}$ \\
\hline Brassica rapa $\mathrm{L}$. & Choy sum & Choy sum & & \\
\hline Colocasia esculenta (L.) Schott & Taro leaves & Taro leaves - Atiefaro & & $\mathrm{X}$ \\
\hline Colocasia esculenta (L.) Schott & Taro leaves & Taro leaves - Faro & & \\
\hline Colocasia esculenta (L.) Schott & Taro leaves & Taro leaves - Omu & & $\mathrm{X}$ \\
\hline Cucurbita maxima Duch ex Lam. & Pumpkin leaves & Pumpkin leaves & & $\mathrm{X}$ \\
\hline Cyathea hornei (Baker) Copel & Fern & Savita & & \\
\hline Diplazium esculentum Swartz & Fern & Puha & & \\
\hline
\end{tabular}


Table 6 (continued)

\begin{tabular}{|c|c|c|c|c|}
\hline Crop species & Common English Name & Local name & Description & $\begin{array}{l}\text { Consumed in } \\
24 \mathrm{~h} \mathrm{MPR}\end{array}$ \\
\hline Diplazium spp & Fern & Fengo & & $\mathrm{X}$ \\
\hline Diplazium spp. & Fern & Unofengo & & $\mathrm{X}$ \\
\hline Ficus copiosa Steud. & Sandpaper Cabbage/Plentiful Fig & Inomahi & & $\mathrm{X}$ \\
\hline Ipomoea aquatica Forsk. & Kang kong & KanKung & & $\mathrm{X}$ \\
\hline Polyscias fruticosa (L.) Harms & Bebero/Geke/Tagala & Wagozo & Yellow leaf & \\
\hline Polyscias fruticosa (L.) Harms & Bebero/Geke/Tagala & Wagozo & Ringiti (thin serated leaves) & \\
\hline Polyscias verticillata Stone & Bebero/Geke/Tagala & Wagozo & Wide leaf & \\
\hline $\begin{array}{l}\text { Rorippa nasturtium-aquaticum (L.) } \\
\text { Hayek }\end{array}$ & Watercress & Watercress & & $\mathrm{X}$ \\
\hline Sauropus androgynus (L.) Merr & Sweet Leaf & Bonio - Red bottom & & \\
\hline Sauropus androgynus (L.) Merr & Sweet Leaf & Bonio - White bottom & & \\
\hline Stenochlaena palustris (Burm.f.) Bedd & Fern & Rosi & & \\
\hline unidentified wild plant & unidentified wild plant & $\mathrm{Bie}$ & wide leaf, wild & \\
\hline Xanthosoma sagittifolium (L.) Schott & Chinese Taro leaves & Karuvera leaves & & $\mathrm{X}$ \\
\hline \multicolumn{5}{|l|}{ Other Vegetables } \\
\hline Cucumis sativus L. & Cucumber & Cucumber & Green skin, white flesh & $\mathrm{X}$ \\
\hline Cucumis sativus L. & Cucumber & Cucumber & White skin and flesh & $\mathrm{X}$ \\
\hline Solanum melongena $\mathrm{L}$. & Eggplant & Eggplant & White striped & $\mathrm{X}$ \\
\hline Zea mays $L$ & Corn/Maize & Corn & Yellow flesh & $\mathrm{X}$ \\
\hline \multicolumn{5}{|l|}{ Nuts } \\
\hline Canarium salomonense B.L.Burtt & Canarium nut & Ngali nut & Itofo & $\mathrm{X}$ \\
\hline Canarium indicum $\mathrm{L}$. & Java/Canarium nut & Ngali nut & Big & $\mathrm{X}$ \\
\hline Canarium harveyi Seem & Canarium nut & Reef nut & & \\
\hline Canarium indicum $\mathrm{L}$. & Canarium nut & Gasio (black nut) & & \\
\hline Barringtonia edulis Seem. & Cut nut & Voze voze & White flesh & $\mathrm{X}$ \\
\hline Barringtonia novae-hibernae Laut. & Cut nut & Anire tinge & White flesh & \\
\hline Barringtonia novae-hibernae Laut. & Cut nut & Cut nut - longer fruit & White flesh & $\mathrm{X}$ \\
\hline Barringtonia novae-hibernae Laut. & Cut nut & Cut nut - purple inside & White flesh & \\
\hline Barringtonia procera $\mathrm{R}$ Knuth & Cut nut & $\begin{array}{l}\text { Green tinge (skin } \\
\text { colour) }\end{array}$ & White flesh & $\mathrm{X}$ \\
\hline Barringtonia procera $\mathrm{R}$ Knuth & Cut nut & $\begin{array}{l}\text { Purple tinge (skin } \\
\text { colour) }\end{array}$ & White flesh & \\
\hline \multicolumn{5}{|l|}{ Animals } \\
\hline Anas superciliosa Gmelin, 1789 & Duck & Duck & & \\
\hline Ducula rubricera (Bonaparte, 1854) & Red knobbed Fruit Pigeon & Kurukuru & & \\
\hline Egretta sacra (Gmelin, 1789) & Pacific reef heron & Hou & & \\
\hline Fregata minor (Gmelin, 1789) & Great Frigate Bird & Belama & & \\
\hline Gallus gallus (Linnaeus, 1758) & Chicken & Chicken (whiteman) & & $\mathrm{X}$ \\
\hline Gallus gallus (Linnaeus, 1758) & Naked Neck Chicken & $\begin{array}{l}\text { Chicken (featherless } \\
\text { neck) }\end{array}$ & & \\
\hline Larus spp. & Seagull & Helekai & & \\
\hline Phalangeriforme spp. & Possum & Possum & & \\
\hline Porphyrio porphyrio (Linnaeus, 1758) & Purple Swamphen & Red nose bird (Bichere) & & \\
\hline Psittaciformes spp. & Parrot & Parrot & & \\
\hline Pteropus vampyrus (Linnaeus, 1758) & Flying fox & Flying fox & & \\
\hline Rhyticeros plicatus (J. R. Forster, 1781) & Hornbill & Hornbill & & \\
\hline Sus scrofa domesticus Erxleben, 1777 & Pig & Pig (crossbreed) & & \\
\hline Sus scrofa Linnaeus, 1758 & Pig & Pig (wild) & & \\
\hline \multicolumn{5}{|l|}{ Seafood } \\
\hline Acanthurus lineatus (Linnaeus, 1758) & Lined surgeon fish & Bireke & & $\mathrm{X}$ \\
\hline
\end{tabular}


Table 6 (continued)

\begin{tabular}{|c|c|c|c|c|}
\hline Crop species & Common English Name & Local name & Description & $\begin{array}{l}\text { Consumed in } \\
24 \mathrm{~h} \mathrm{MPR}\end{array}$ \\
\hline $\begin{array}{l}\text { Acanthurus nigricauda Duncker \& Mohr, } \\
1929\end{array}$ & Epaulette surgeonfish & Evaeva & & \\
\hline Acanthurus nigrofuscus (Forsskål, 1775) & Brown Tang (Surgeon fish) & Asirae & & \\
\hline $\begin{array}{l}\text { Acanthurus xanthopterus Valenciennes, } \\
1835\end{array}$ & Yellow fin Surgeon fish & Tavazi & & \\
\hline Anadara antiquata (Linnaeus, 1758) & Antique Ark (Bivalve) & Deo & & \\
\hline $\begin{array}{l}\text { Anguilla marmorata Quoy \& Gaimard, } \\
1824\end{array}$ & Giant mottled eel & Eelfish & & \\
\hline Atactodea striata (Gmelin, 1791) & Striate beach clam & Kenekene & & \\
\hline Balistidae & Triggerfish & Fubua & & \\
\hline Balistoides viridescens & Titan triggerfish & Makoto & & \\
\hline Birgus latro (Linnaeus, 1767) & Coconut crab & Coconut crab & & $\mathrm{X}$ \\
\hline $\begin{array}{l}\text { Bolbometopon muricatum (Valenciennes, } \\
1840 \text { ) }\end{array}$ & Humphead Parrot fish & Topa & & \\
\hline Cambarus spp. & Freshwater lobster & Crayfish & & \\
\hline Caranx spp. & Trevally & Mamula & & $\mathrm{X}$ \\
\hline Caulerpa lentillifera (J.G. Agardh, 1837) & Sea grapes & Seaweed & & \\
\hline $\begin{array}{l}\text { Cerithidea quadrata G. B. Sowerby II, } \\
1866\end{array}$ & Black chut-chut & Ropi & & \\
\hline Chelonia mydas (Linnaeus, 1758) & Green sea turtle & Sea turtle & & $\mathrm{X}$ \\
\hline Dermochelys coriacea (Vandelli, 1761) & Leatherback turtle & Turtle & & \\
\hline $\begin{array}{l}\text { Elagatis bipinnulata (Quoy \& Gaimard, } \\
\text { 1825) }\end{array}$ & Rainbow runner & Rainbow (Babalu) & & \\
\hline Encrasicholina punctifer Fowler, 1938 & Buccaneer anchovy & Eoea & & \\
\hline Epinephelus hexagonatus (Forster, 1801) & Starspotted grouper & Orufu & & $\mathrm{X}$ \\
\hline Epinephelus lanceolatus (Bloch, 1790) & Giant Grouper & Zoata & & \\
\hline Epinephelus spp & Round head grouper & Bukulu & & \\
\hline Etelis spp. & Deep water snapper & Noto & & \\
\hline Gazza achlamys Jordan \& Starks, 1917 & Smalltoothed ponyfish & Sogari & & \\
\hline $\begin{array}{l}\text { Halichoeres argus (Bloch \& Schneider, } \\
\text { 1801) }\end{array}$ & Angus wrasse (Very slippery) & Zaoto & & \\
\hline Istiophoridae & Marlin & Viviru & & \\
\hline Katsuwonus pelamis (Linnaeus, 1758) & Skipjack Tuna & Bonito & & $\mathrm{X}$ \\
\hline Kuhlia marginata (Cuvier, 1829) & $\begin{array}{l}\text { Dark-margined flagtail (River } \\
\text { fish) }\end{array}$ & Hegosune & & \\
\hline Lethrinus miniatus (Forster, 1801) & Sweetlip emperor & Mihu & & \\
\hline Macrobrachium lar (J.C.Fabricius, 1798) & Prawn/shrimp & Prawn & Freshwaster & \\
\hline Myripristis spp. & Soldier fish & Fufu & & $\mathrm{X}$ \\
\hline Naso brevirostris (Cuvier, 1829) & Canvass or Unicorn fish & Fagu & & \\
\hline Octopus cyanea Gray, 1849 & Octopus & Octopus & & \\
\hline Philypnodon grandiceps (Krefft, 1864) & Olive flat head gudgeon & Begozo & & \\
\hline Pinctada margaritifera (Linnaeus, 1758) & Oyster & Riki & & $\mathrm{X}$ \\
\hline Plectorhinchus lineatus (Linnaeus, 1758) & Yellowbanded sweetlips & Fehu & & \\
\hline $\begin{array}{l}\text { Pseudomyxus capensis (Valenciennes, } \\
\text { 1836) }\end{array}$ & Freshwater mullet & Embo & & \\
\hline Sardinella spp. & Sardines & Katukatu & & \\
\hline Sargocentron tiereoides (Bleeker, 1853) & Pink Squirrel fish & Heta & & $\mathrm{X}$ \\
\hline Scaridae or Scarus spp. & Parrot fish & sioura & & \\
\hline Scomberoides lysan (Forsskål, 1775) & Doublespotted queenfish & Eusava, lasilasi & & \\
\hline Scomberomorus cavalla (Cuvier, 1829) & Spanish mackerel & Kingfish & & \\
\hline Scylla serrata (Forskål, 1775) & Mud crab & Kapehe & & $\mathrm{X}$ \\
\hline Sepioteuthis lessoniana d'Orbigny, 1826 & Reef Squid & Squid & & \\
\hline
\end{tabular}


Table 6 (continued)

\begin{tabular}{|c|c|c|c|c|}
\hline Crop species & Common English Name & Local name & Description & $\begin{array}{l}\text { Consumed in } \\
24 \mathrm{~h} \mathrm{MPR}\end{array}$ \\
\hline Siganus corallinus (Valenciennes, 1835) & $\begin{array}{l}\text { Blue-spotted spinefoot (Yellow } \\
\text { reef fish) }\end{array}$ & Gore & & $\mathrm{X}$ \\
\hline Siganus lineatus (Valenciennes, 1835) & Golden-lined spinefoot & Sirusiru & & \\
\hline Sphyraena barracuda (Edwards, 1771) & Pinkhandle or Obtuse Barracuda & Gohi & & $\mathrm{X}$ \\
\hline Thunnus albacares (Bonnaterre, 1788) & Yellowfin tuna & Tatalingi & & $\mathrm{X}$ \\
\hline Toxotes jaculatrix (Pallas, 1767) & Archer fish & Vavanaka & & \\
\hline Trachinotus baillonii (Lacepède, 1801) & Small spotted dart & Dalo & & \\
\hline $\begin{array}{l}\text { Tylosurus crocodilus (Péron \& Lesueur, } \\
\text { 1821) }\end{array}$ & Houndfish or Needlefish & Somasoma & & \\
\hline \multicolumn{5}{|l|}{ Egg } \\
\hline Anas superciliosa Gmelin, 1789 & Egg & Duck egg & & \\
\hline Birgus latro (Linnaeus, 1767) & Egg & Coconut Crab egg & & \\
\hline Chelonia mydas (Linnaeus, 1758) & Egg & Turtle egg & & \\
\hline Dermochelys coriacea (Vandelli, 1761) & Egg & Leathback turtle egg & & $\mathrm{X}$ \\
\hline Ducula pistrinaria Bonaparte, 1855 & Egg & Dove egg & & \\
\hline Ducula rubricera (Bonaparte, 1854) & Egg & Dove egg & & \\
\hline Gallus gallus (Linnaeus, 1758) & Egg & Chicken egg & & $\mathrm{X}$ \\
\hline Megapodius eremita Hartlaub, 1868 & Egg & Megapode egg & & \\
\hline Panulirus penicillatus (Olivier, 1791) & Egg & Crayfish egg & common & \\
\hline Porphyrio porphyrio (Linnaeus, 1758) & Egg & Rednose Bird egg & & \\
\hline Unidentified & Egg & Fish egg & & \\
\hline Unidentified & Egg & Punder egg & & \\
\hline
\end{tabular}

from the focus group shared: "Before, we use to eat more local foods such as taro, yam, pana, and banana. Now, more of the foods in our diet are processed because they are easy to get and very fast to cook." The most recent and reportedly profound shift was due to tree loggers working near Baniata in the 1990s, introducing the villagers to even more imported foods than before. Another man agreed, adding, "Villagers are no longer as interested in local foods because they take time to cook, unlike processed foods [which] are easier to cook and require less firewood."

Local villagers were beginning to experience increased rates of NCDs such as heart disease, high blood pressure, and diabetes. According to one woman in the focus group: "Diet changes that are currently happening will result in unhealthy people in the village, since now we start to see some people are sick and not strong. There is an increase in shortness of breath, belly aches, muscle weakness, and sick feelings from eating processed foods."

\subsubsection{Food system changes}

According to village elders, Baniata's traditional and current agricultural system is entirely organic, as it does not rely on any external agri-chemical inputs (synthetic fertilizers, pesticides, herbicides, and fungicides). However, pests and diseases are reported to be increasing, including rat infestations. Garden productivity was perceived to be decreasing due to climate change, pests, increased land pressures, and decreased fallowing times. Climate changes identified include increasingly inconsistent seasonal weather patterns and more intense periods of heavy rains. These weather changes are challenging Indigenous agricultural practices which have been utilised for centuries.

Climate change related weather patterns were described by the villagers as becoming "more intense" and "more frequent", adding increased stress to food production. One villager noted, "since the 1990s, more frequent natural disasters occur". Villagers have previously understood weather patterns and prepared for cyclones around December. One man stated, "now, cyclones are much more difficult to predict". Stronger storms and heavier rains are causing increased flooding that "destroys gardens" and "creates landslides that block rivers". Villagers also reported that increased rains are washing away soils and giving rise to increases in crop pests and diseases. Knowledge regarding planting seasons, fishing patterns, and crop harvests are being challenged. Water quality and security was reported as a non-issue.

\subsubsection{Economic and social development}

Household incomes were predominantly generated through the sale of agri-food products at regional produce markets in the towns of Munda and Noro. All villagers sell agri-food 
Table 7 Knowledge, attitudes and practices (KAP) survey results from Baniata Village, Solomon Islands ( $\mathrm{n}=30$ women)

\begin{tabular}{|c|c|c|c|}
\hline Knowledge & 2 correct responses & 1 correct response & No correct response \\
\hline Can you identify two health issues from being overweight or obese? & $60 \%$ & $26.6 \%$ & $13.3 \%$ \\
\hline Can you share two reasons why someone might be overweight? & $56.6 \%$ & $26.6 \%$ & $13.3 \%$ \\
\hline Can you name two benefits of eating fruit? & $39.8 \%$ & $50.0 \%$ & $10.2 \%$ \\
\hline Can you name two foods rich in Vitamin A? & $33.3 \%$ & $63.3 \%$ & $3.3 \%$ \\
\hline Attitudes & Disagree & Neutral & Agree \\
\hline I am satisfied with my food choices & $6.7 \%$ & $3.3 \%$ & $90 \%$ \\
\hline Fruit and vegetables are expensive to purchase & $43.3 \%$ & $16.7 \%$ & $40 \%$ \\
\hline It's important to prepare a wide variety of foods & $6.7 \%$ & $3.3 \%$ & $90 \%$ \\
\hline It's challenging to prepare a wide variety of foods & $40.4 \%$ & $17.2 \%$ & $42.4 \%$ \\
\hline It is important to provide fruits and vegetables for my family & $6.7 \%$ & $0 \%$ & $93.3 \%$ \\
\hline It is important to provide meat for my family & $23.3 \%$ & $36.7 \%$ & $40 \%$ \\
\hline It is important to provide many fish for my family & $3.3 \%$ & $26.7 \%$ & $70 \%$ \\
\hline It is difficult to get my children to eat fruits and vegetables & $60 \%$ & $10 \%$ & $30 \%$ \\
\hline \multicolumn{4}{|l|}{ Practices } \\
\hline \multirow[t]{2}{*}{ Do you read nutrition facts labels? } & Yes & Sometimes & Never \\
\hline & $3.3 \%$ & $53.5 \%$ & $43.3 \%$ \\
\hline Do you practice food preservation techniques? & Drying & Smoking & None \\
\hline (can provide more than one response) & $86.6 \%$ & $23.3 \%$ & $13.3 \%$ \\
\hline
\end{tabular}

products for consumption and sale at markets. Accessing these markets requires a 90-min petrol-powered commute by sea from Baniata. Travel time in combination with year-round humidity and high temperatures $\left(\sim 30^{\circ} \mathrm{C}\right)$ creates quality challenges for agri-food market sales. Primary agri-food products for sale at local and regional markets included homegrown yams, cassava, taro, sweet potatoes, bananas, fruits, green leafy vegetables, nuts, and less frequently - fresh fish. Two agri-food products were also sold in larger quantities in national or international markets: copra (dried coconut) used to produce coconut oil and locally processed dried ngali nuts (Canarium indicum L.).

Baniata villagers identified several barriers to market access and income generation. The cost of petrol is high in relation to incomes, creating an economic barrier to market access given the amount of fuel required to access the central market. Market access is limited during certain seasons due to the small size of the boat used and rough seas. The lack of adequate storage and refrigeration causes agri-food products to lose quality in the hot, humid environment; both during the commute and throughout the day at the markets. Lastly, higher numbers of vendors are selling their goods at the markets, creating a larger supply causing lower prices and sales. However, the women reached consensus that most families were generating enough income to provide for essential daily activities, which include purchasing food, goods and housing materials from outside of the community, and children's school fees. Trading of goods between communities and villagers was also reported, but this tradition is declining as cash is increasingly prioritized.

\subsubsection{Food and nutrition security}

Men and women agreed, as incomes rise so does the purchasing of imported foods from shops outside of Baniata. One female focus group participant shared that "traditional crops are increasingly sold for [store-bought] items, [such as] white rice, sugar, biscuits, soap (for body and clothes), and salt”. Disagreement existed between the men and women on how quickly the nutrition transition was occurring, however both men and women shared fears that their Indigenous food system is being threatened.

Seasonal fluctuations in food availability limits the number of foods available during certain periods of the year. In periods of low food availability, imported rice is an increasingly preferred food source due to its convenience, taste and low cost. However, one woman stated, "rice does not keep us full as long as kumara (sweet potato; Ipomoea batatas), and we get hungrier more quickly when working in the gardens".

Seafood catches were also reported to be in decline. Men stated that "fish is declining as it is not easy to catch fish nowadays, indicated by the length of time to go fishing, distance to walk or paddle to do fishing". Older men reported fewer fresh fish in their diets from when they were younger. Another man added "even in the rivers, there used to be plenty of fish and eels. Nowadays both fish and eels have declined, 
Fig. 2 Four dietary diversity and diet quality indicators compared with body fat percentages in Baniata Village, Solomon Islands ( $n=30$ women). The four diet diversity indicators used for comparison with body fat percentage were: dietary diversity score (DDS), food variety score (FVS), mean adequacy ratio (MAR), dietary species richness (DSR)
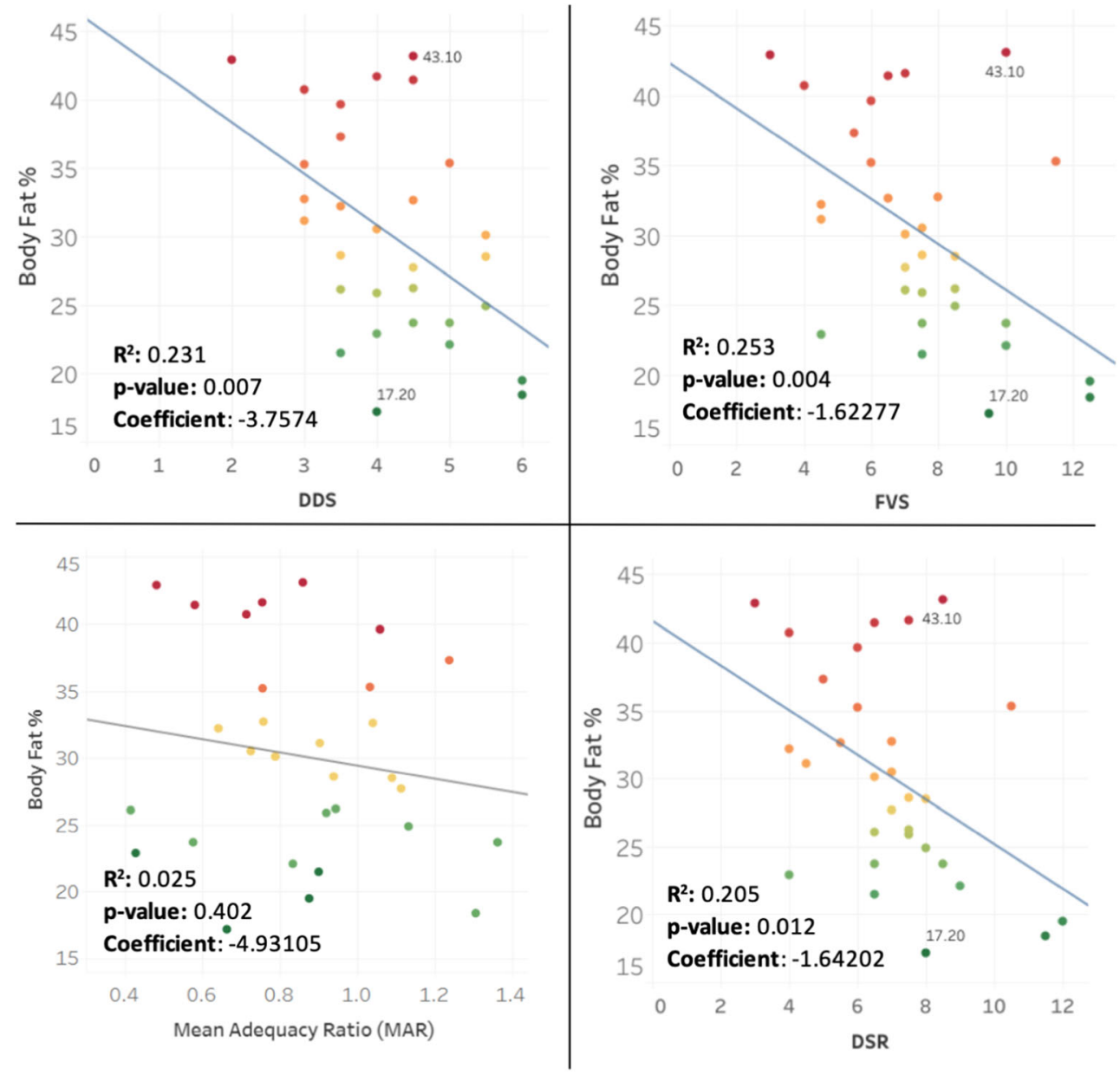

probably due to increased human population as well as flooding that washed the eels out to the sea".

\subsubsection{Utilization of local agrobiodiversity}

Collectively, men and women identified 221 species and varieties that were previously or currently available for use as foods within the local landscape. These foods were categorized by homegrown and market foods $(63 \%)$ or wild foods $(37 \%)$. Of these species and varieties 67 were roots, tubers, or bananas, 51 seafood, 26 dark green leafy vegetables, 25 fruits and vegetables, 16 legumes, 14 animals, 12 eggs, and 10 nuts and seeds (Fig. 4, Table 6).
Fig. 3 Food system transition timeline from focus group discussions in Baniata Village, Solomon Islands

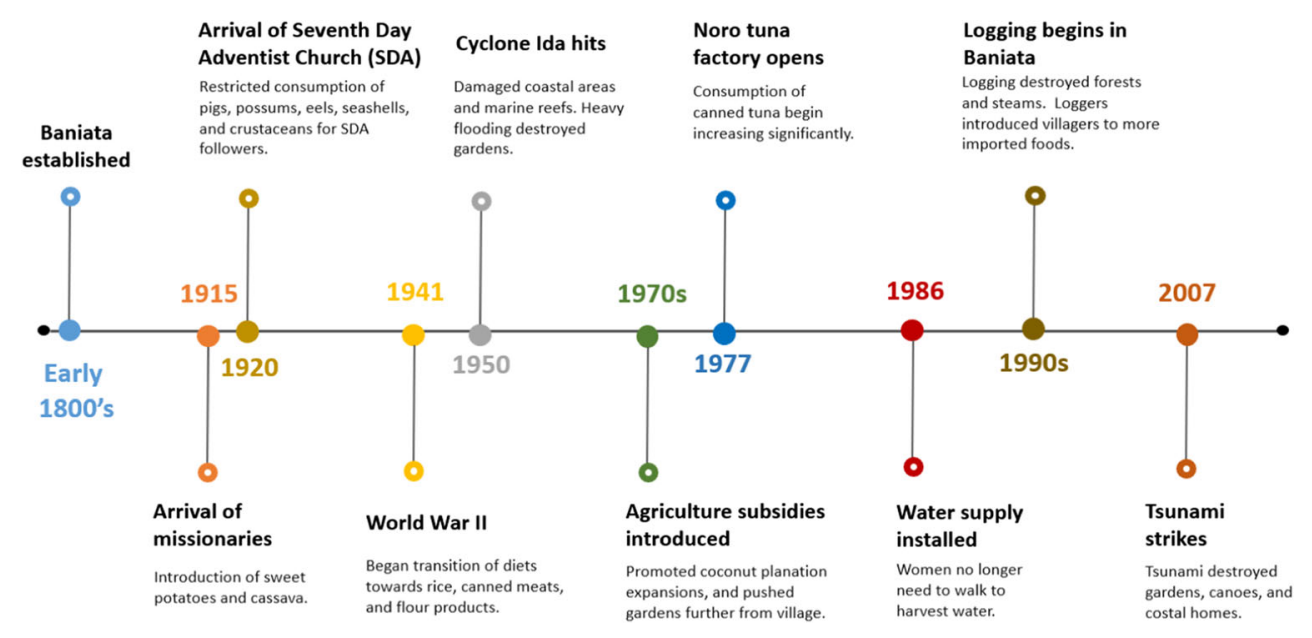


Focus group participants came to the consensus that traditional knowledge and utilization of local agrobiodiversity is rapidly declining. Both men and women focus groups outlined how traditional language, hunting and fishing techniques, food preparation traditions, and utilization of local agri-food biodiversity is being lost due to the increased reliance and consumption of imported foods. "Foods in the past are very good and healthy and make the body stronger but now, local foods are not always eaten and our health is decreasing compared to the past."

The transferring of traditional knowledge is exclusively an oral custom, which typically occurs during gardening and cooking for women, and during hunting and fishing for men. Village elders used to teach young boys how to hunt and fish, and while doing so, they share stories, traditions, and biodiversity utilization knowledge. Similarly, women would teach girls how to garden, prepare meals, and conduct household chores. Both groups of men and women were largely convinced that their children are uninterested in learning the traditional ways of life, stating that children view modern lifestyles as being more appealing. Contradicting these views, the majority of adolescent and children's FGD participants (94\%), expressed a strong interest in continuing traditions such as gardening, fishing, collecting wild foods, and raising a family in the village.

While there is interest by both the men and women of Baniata to preserve traditional foods and recipes, when prompted about the future of their food system, villagers projected that "in 20-year time, we might still eat our own kind [offood] since we are still practising how to collect them, but not frequent like now unless we start doing something to look after our local varieties and breeds".

\subsubsection{Youth food system perspectives}

Youth FGDs explored the role of boys and girls in the local food system, their dietary preferences, and aspirations for the future. Boys still took part in hunting and fishing with their fathers, whereas girls helped garden and cook. When asked about the future, most of the youth ( $82 \%$ ) expressed interest in taking over the family farm when they get older. The minority $(18 \%)$ wanted to leave the village to attend university and/or get married, and then eventually return to Baniata.

When prompted, youth were able to differentiate between traditional and imported foods. The majority of youth participants shared their enjoyment towards eating traditional foods, their favourites being mangos, cabbage, bananas, jackfruit, coconuts, paw paws, ngali nuts. Many also expressed their affinity towards imported foods such as white rice, noodles, cheese puffs, and sweetened biscuits. Looking towards the future, most $(75 \%)$ agreed that they wanted to continue eating traditional foods as well as imported foods.

\section{Discussion}

Our study examined dietary intakes among Indigenous Solomon Islanders in a remote rural community using repeat $24 \mathrm{~h}$ MPRs and found dietary intakes of several essential nutrients to be insufficient. Additionally, macronutrients were unbalanced when compared to the recommended macronutrient range as a percent of total energy. Energy intakes included large amount of fat (primarily from coconut products), which was overconsumed by over three quarters of women. Saturated fat intakes exceeded recommendations by $70 \%$. Whilst fat intakes are an important contributor to maintaining recommended energy intakes within the lean seasons, these are likely habitual fat intakes throughout the year which is attributing to the overweight and obesity rates. More than half $(53 \%)$ of women had protein intakes below EAR, despite most women consuming some form of fish or seafood. Other sources of protein such as meat, poultry, eggs and legumes were not widely consumed. Fibre intakes were below recommendations, likely attributed to the increasing trend of substituting traditional staples of roots and tubers high in fibre, with low fibre white refined rice, together with low of fruit and vegetable consumption.

Several micronutrients were inadequately consumed. The majority of women had inadequate intakes of calcium (97\%) and zinc (40\%), likely linked to the limited quantities of animal source foods consumed, exasperated by low consumption and diversity of the diet. Dairy products which are high in calcium are not traditionally part of the diet, nor were they consumed. Promotion of local foods high in calcium, such as dark green leafy vegetable, could help improve calcium and overall micronutrient intakes. However, it is important to note, that over two-thirds of participants reported betelnut use, which is common practice in Solomon Islands and across the Pacific. Betelnut is combined with ground reef coral (calcium carbonate; $\mathrm{CaCO} 3$ ) to produce an intoxication effect. Coral may supply dietary calcium, however betelnut is classified as a Group 1 carcinogen by the International Agency for Cancer Research, and its use should not be encouraged. Cases of hypercalcemia have been reported from heavy users of betelnut (Lin et al., 2002). Insufficient vitamin A intakes were likely due to insufficient intake of foods rich in beta-carotene such as papaya, mango, Fe'i banana and orange sweet potatoes despite these being available. Another contributing factor is also likely the transition away from the catching and consumption of wild small fish, traditionally consumed whole, which contain high quantities of vitamin $\mathrm{A}$ in the eyes and organs, as well as calcium in the bones.

Participants who consumed higher number of plant and animal species had a significantly higher probability of meeting nutrient recommendations (MAR) and a lower body fat percentage. The mechanism behind lower body fat percentages may involve a substitution effect by displacing energy- 
Fig. 4 Available agrobiodiversity organised by food group in Baniata Village, Solomon Islands $(n=221)$

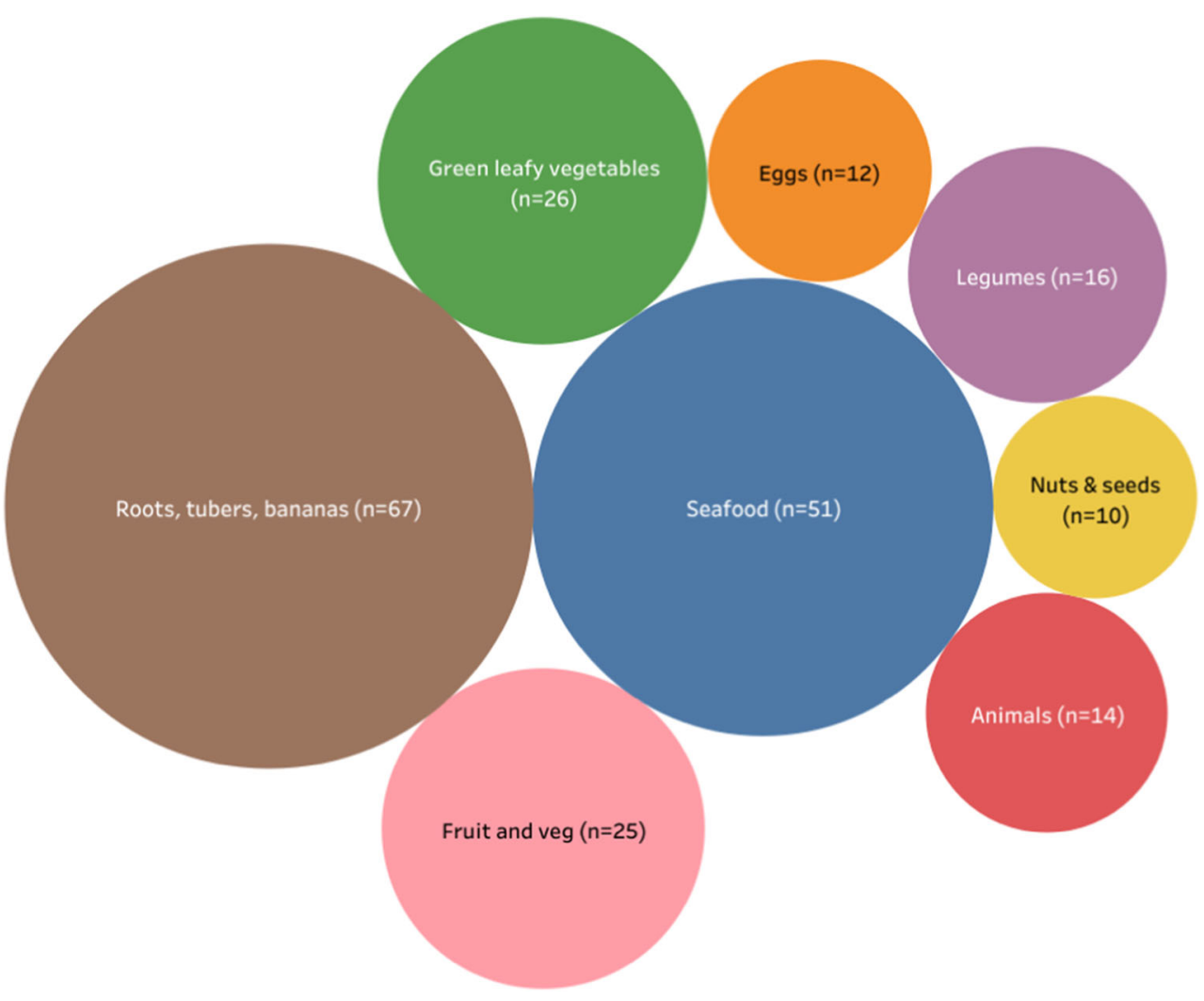

dense and nutrient-poor ultra-processed foods with increased healthy and diverse foods (Poti et al., 2017). Additionally, consuming more fruits and vegetables has been associated with improved health outcomes including lower body fat percentages (He et al., 2004). Evidence is clear that higher body fat percentages are linked to increased cardiovascular disease and other NCD risks (Lee et al., 2011). Variability between species diversity and body fat percentage were high, but significant. Though the sample size was small, our findings provide some evidence that consuming an increased diversity of minimally processed species may contribute to a lower body fat percentage (Asghari et al., 2017). However, ensuring macronutrient and energy balances are important considerations when seeking to scale up dietary diversity. The positive relationship between diet quality and body fat has been supported in the literature for certain populations, but not yet in those living in the Pacific (Jayawardena et al., 2013). Rapidly increasing rates of overweight and obesity are critical concerns driving the NCD epidemic across the Pacific, and the current messages of consume less of foods higher in salt, sugar and fat and increase exercise alone are not working. To the authors knowledge, this is the first study to demonstrate a positive relationship between higher levels of food biodiversity in the diet and lower body fat percentage. Adjusting for energy intake did not significantly change these associations (see supplementary information). High body fat is a known risk factor for heart disease, hypertension, stroke, diabetes mellitus, and various forms of cancer (Nishida et al., 2010). Our findings suggest a potential linkage between the consumption a wider variety of traditional, agrobiodiverse foods and a lower body fat percentage.

The nutrition transition is a complex issue with economic, political, physical activity and dietary drivers. Our study reported dietary changes to start during WW2, a global trend that has been reported elsewhere (Barry M. Popkin, 2015). Nutrition transition categories can be identified through 5 stages, of which Solomon Islands appears to be at stage $4-$ characterized by decreasing physical activity and westernization of diets leads to increased NCD prevalence (Popkin \& Gordon-Larsen, 2004). Overweight and obesity rates have been increasing across the Solomon Island population, for children, adolescences and adults (UNICEF/WHO, 2019). Diabetes prevalence has also been steadily increasing (UNICEF/WHO, 2019). Dietary risk factors are associated with high rates of burden of disease in the Solomon Islands (Afshin et al., 2019). FGD participants reported qualitatively that diets have been changing, moving away from traditional foods to more modern and western style foods - and simultaneously health status has deteriorated in regard to NCD prevalence. Our dietary intake data, while cross-sectional also presents evidence that modern and increasingly processed foods are an important part of the diet and contribute significantly to overall energy intake and increasing rates of overweight and obesity. Together with the high BMIs and body fat percentage 
documented, these data confirm other documented trends of increasing NCDs that the nutrition transition is underway in Solomon Islands.

FGD participants shared key historical and climatic narratives perceived to be drivers of a nutrition transition. During these discussions, participants identified 221 species and varieties of agrobiodiverse foods available and explained that many varieties are decreasing due to changing demands and preferences towards imported and ultra-processed foods. Participants expressed their concern for the loss of traditional knowledge and food varieties within their Indigenous food system. These perceived nutrition transitions are wellsupported by literature where data show processed foods in the Pacific are accelerating, predominantly in low- and middle-income countries, including Solomon Islands. (Santos et al., 2019; Sievert et al., 2019; Thow et al., 2011). Implications of imported processed foods should be further examined with considerations to convenience, affordability, nutrient contributions, and food security.

\subsection{Dietary quality and composition}

This is the first recent study to examine quantitative dietary intakes among Indigenous Solomon Islanders solely in a rural remote community, and reflect the findings of other dietary assessments conducted in PSIDS (Haddad et al., 2015; Horsey et al., 2019; Hughes \& Lawrence, 2005; Konishi et al., 2011). Recent regional data indicate a re-emergence of the nutritional deficiency beriberi (related to vitamin $\mathrm{B}_{1}$ deficiencies) in the neighboring Pacific island of Kiribati, which has not been seen in over five decades (Nilles et al., 2018). Our data indicates that over three-fourths of women consumed less vitamin $\mathrm{B}_{1}$ than the EAR, which could be related to the reported increase in consumption of processed foods, in particular of refined white rice, replacing a diverse range of previously utilised traditional foods rich in B vitamins.

Despite having access to a wide variety of traditional biodiverse foods, consumption of food groups were found to be unbalanced. A recent study in Solomon Islands found slightly higher levels of diet diversity, however different food groups classifications were used, which make comparisons difficult (Horsey et al., 2019). Their study population size was much larger and included a key urban center and trading hub, whereas our study focused on a very remote rural community. Horsey et al. (2019) identified that local food preferences were towards diverse diets, similar to what we found through our FGD discussions, as well as through the KAP study. This suggests that the drivers of food choice are more likely to do with situational contexts such as household food security, incomes, production limitations, and climate change.

Coconuts are a culturally-rich traditional food in Solomon Islands. Coconut plantations were subsidised in the 1970's as a way to promote economic opportunities. These subsidies likely influenced dietary transitions within this study population, which comes with tradeoffs. Coconuts are a year-round, low-maintenance, and climate-resilient source of energy and contributed to over half of participant's saturated fatty acids intakes. Our results indicate that over two-thirds of participants were consuming more saturated fatty acids than the recommended upper limit of $<10 \%$ of total $\mathrm{kJ}$ (FAO/WHO, 2010). A recent meta-analysis of 16 clinical trials concluded that coconut fat should not be viewed as healthy when consumed in large quantities in relation to cardiovascular disease risk. (Neelakantan et al., 2020). Saturated fat-rich diets are more obesogenic than diets with lower saturated fat contents (Hariri et al., 2010). Therefore, diets excessively high in coconuts, coupled with increased consumption of saturated fats and ultra-processed foods, may be compounding variables contributing to the rise of NCDs in Pacific populations.

Imported foods contributed the second highest source of energy (kJ) (34.1\%) after homegrown garden foods, including both NOVA $1-3$ and NOVA 4 categories $(22.8 \%$ and $11.3 \%$ respectively). NOVA 4 category ultra-processed foods are strongly associated with increased rates of NCDs including obesity, cardiovascular disease and cancers (Monteiro et al., 2019). Most published studies that capture ultra-processed foods in the diet come from upper-middle and upper income countries and report higher percentages of ultra-processed foods in the diets, from 15 to $48 \%$ (Baker \& Friel, 2016; Moubarac et al., 2017). Our study showed that intakes of these ultra-processed foods were relatively low in comparison, not unexpected considering the remoteness of the population. Considering our study was conducted in the lean season, these ultra-processed foods contributed to ensuring populations maintained adequate energy. However, the consumption of these foods are on the rise in Solomon Islands and the Pacific (Horsey et al., 2019), and for a population already battling high rates of overweight, obesity and NCDs, it is imperative that consumption of unhealthy ultra-processed foods does not increase to rates found in more developed countries.

However, it's important not to romanticize traditional diets. The reality is that most of the Pacific will be unable to be completely self-sufficient and reliant on local foods alone. Imported and processed foods have many characteristics that are appealing to consumers. They are often packaged and have undergone transformation and processing that extends their shelf life and decreases cost of production at scale, helping to improve access and close the energy gap in Solomon Islands, particularly in times of high food insecurity. Processed foods are also often preferred as they are quicker to prepare and require less fuel to cook than traditional staple crops, and hence are less of a time burden for women (who are mainly responsible for food preparation and collection of firewood). They also are reported to have a preferred taste, often 
associated with the high levels of salt, sugar and/or fat in them, together with western diet 'aspiration' and the local perception of these foods being modern and 'non-poor'. However, the increased consumption of imported and increasingly ultraprocessed foods displace more micronutrient dense foods (such as roots, tubers, and bananas which are also culturally significant) have other trade-offs on other aspects of food and nutrition insecurity related to negatively affecting diet quality and health (Monteiro et al., 2019). The key is finding the balance between convenience, desirability, health and nutrition.

While the calorie gap has closed with the transitioning food system, dietary gaps for micronutrients and protein remain. Baniata is a coastal village, one that has traditionally relied on fishing as a source of protein. However as reported in the FGDS, wild fish catches are declining due to reported decreases in populations and rises in unpredictable weather patterns, challenging traditional sourcing practices. Despite the opening of the local Noro tuna cannery in 1977, and the community reporting that tuna access than became easier and more affordable in processed form, consumption of protein continues to be insufficient in Baniata. The ease in accessing tinned tuna (which is usually second grade tuna and flesh only) also would decrease the more traditionally caught small reef fish which are often eaten together whole and contain more micronutrients than consuming the flesh alone (Bell et al., 2019). The reliance on tinned tuna flesh may also be furthered due to reduced access to wild reef fish populations due to overfishing and climate change (Bell et al., 2015, 2018). Tinned tuna is preserved in oil, and various brands of processed tinned tuna have founds to have higher contents of fat and salt than what would be with consumption of locally caught fresh fish (Snowdon et al., 2013). Foods such as tayio (canned tuna) are locally processed, high in essential micronutrients, low in mercury, and are shelf stable (Bell et al., 2019). Locally produced canned tuna provides a culturally important and protein-rich processed food for Indigenous Solomon Islanders, and should continue to be promoted as a way to support healthy and resilient food systems for the future. There is an opportunity to create and promote similar processed and shelf-stable foods rooted in traditional knowledge, which contain essential nutrients, take less time to prepare, and meet the cultural and taste preferences of Pacific populations. These foods would have the added benefits of contributing to a stronger local economy by providing improved livelihoods for farmers and sellers of these foods.

\subsection{Contribution of local agrobiodiversity to diets}

Results from our qualitative FDG data indicate that Baniata's Indigenous food system is rich in agrobiodiversity, that has traditionally supported the local food system. However, historical perspectives from both men and women indicate that the Indigenous food system is shifting away from traditional foods towards imported and processed foods related by external influences, convenience, and taste. The influence of loggers and missionaries highlighted in the FGDs is well documented in other contexts within the country and wider Pacific region (Pollock, 2017).

Intentionally cultivated homegrown foods were the primary source of energy (51.7\%), and were primarily sourced from coconut products, starchy root vegetables (sweet potato, taro, yam), and bananas. Wild foods contributed some essential nutrients to local diets; however, community elders and women perceived the collection and consumption of wild foods to be declining. This was surprising, given that coastal Pacific Island communities have traditionally relied on wild fish sources as major contributions to their diet. Given that Baniata is set both on the coast, and on the base of mountains, the lack of wild fish, animals and plants was surprising, especially given the remoteness of the community and that Indigenous communities often rely on wild food sources during lean seasons.

With the exception of seafood, animal sourced foods and protein-rich plant foods were minimally consumed, limiting possible sources of dietary protein. SDA religious followers were restricted from eating mollusks, many of which are traditional sources of protein. Eggs were reported to be minimally consumed, similar to other studies in the Pacific. In many contexts, raising chickens is often an effective way to increase consumption of nutritious animal source foods, which can improve diet diversity and over all diet quality. However, chicken husbandry in the Pacific is difficult, due to the humid, tropical conditions. Opportunities for local egg production using traditional rather than hybrid breeds, may offer a solution (Padhi, 2016).

Overall, homegrown foods still dominate the diet, but reported declining food diversity (species and varieties), a heavy reliance on coconut products, and increase in consumption of imported and processed foods are likely contributing to increased rates of overweight and obesity within the region.

\subsection{Food system outlooks}

Our findings indicate that traditional knowledge is not adequately being transmitted from older to younger generations. We found a significant discrepancy between adults and youth when discussing knowledge transmission. Adults felt youth were uninterested in learning traditional knowledge pertaining to food systems, however most of the youth expressed their interest in traditional food varieties and continuing with the family farm as they aged. Finding a way to harness traditional knowledge in a way that appeals to youth, such as through school nutrition education or marketing campaigns, may be a way to document and continue the transferring of Indigenous knowledge. 
The FGDs provided unique insights into climatic and environmental drivers impacting food system sustainability and dietary diversity, such as stronger storms, increases in pests, and fewer fishing catches. Recent literature supports participant claims, as climate change is expected to be a strong contributor to household food insecurity (Wheeler \& von Braun, 2013) by challenging traditional food production and fishing practices (McIver et al., 2016). To build resiliency, innovation is required. Examples include empowering fishermen with more efficient fishing techniques (Asch et al., 2018; Bell et al., 2018), and diversifying food production to supply a wider range of nutritious and climate-resilient crops, such as livestock, legumes, nuts, and seeds (Headey et al., 2018).

Declining agricultural productivity and population growth were both raised as major concerns as threats to the health and livelihoods and food security of Indigenous rural Solomon Islanders. Participants reported little nutrient recycling in the form of manure or food compost to home gardens, which is an exit point for nutrients within the local food system (Mohee et al., 2015; Roos et al., 2016). Poor soil health likely stems from decreased fallowing opportunities and a lack of nutrientrich soil amendments. Similar observations in declining soil health have been made in the Melanesian Highlands of Papua New Guinea (Fujinuma et al., 2018).

\subsection{A case for building a more resilient food system}

Our results indicate that improving the production and consumption of agrobiodiversity within food systems may have a positive impact on nutrition by improving the micronutrient content of local diets, and potentially encouraging a healthier body weight. This builds upon the growing evidence that agrobiodiversity is essential for a sustainable food future (Zimmerer \& Haan, 2017) and improved human nutrition and health, particularly in light of climate change (Zimmerer et al., 2019). In particular, leveraging a diverse range of neglected and underutilised species which are culturally significant, climate-resilient, and contain high levels of nutrients currently missing in local diets could prove particularly effective in improving diet quality and build residency towards climate change (Raneri et al., 2019).

Supporting innovations that identify novel and appealing approaches to promoting nutritious traditional, neglected and underutilied foods with improved cooking technologies may help improve consumption rates (Dweba \& Mearns, 2011), particularly since focus group participants cited enjoyment in the taste of these culturally significant foods. For example, promoting local alternatives to white rice such as golden kumara (beta-carotene rich) provide starchy dietary alternatives, which are less likely to contribute to NCD risk (McLennan \& Ulijaszek, 2015). Loss of wild fish has been reported by villagers, but canned tuna can serve as a traditional and non-perishable strategy for improving protein and nutrient intakes. Legumes were found to be severely underutilised and are of particular interest due to their high protein and fibre content, as well as their unique ability to fix nitrogen in the soil and act as a growth-promoting fertilizer (Masset et al., 2014). With the exception of dried ngali nuts, food preservation techniques were underutilised, posing a food security threat in times of poor harvests or during climatic events. Strengthening the capacity of smallholder women farmers to store, preserve, and package local, nutrient-dense foods is likely to help households achieve better food security yearround (Dweba \& Mearns, 2011) and potentially reduce dependence on processed and refined imported staple foods.

Trade-offs associated with imported foods should be critically examined from nutritional, environmental, economic, and socio-cultural perspectives. Imported and processed foods can play a role in providing affordable and convenient options for families. Previous studies have documented the links between nutrition knowledge and nutritional literacy with better food choices, healthier diets, and healthy body compositions (Michou et al., 2018; Taylor et al., 2019). Promoting nutrition education, particularly if harnessing traditional knowledge, can help build adaptive capacity towards climate change (Granderson, 2017) and improve the nutrient density of food supplies. It is recommended that nutrition education interventions aim at increasing knowledge of local food benefits, as well as highlighting the risks associated with the overconsumption of ultra-processed foods.

Importantly, Indigenous food systems and associated knowledge provides many answers to concerns of modernday industrial food systems. Indigenous foods systems have traditionally provided food and nutrition while maintaining natural resources, the environment, and biodiversity. Scaling up Indigenous knowledge can help inform the global debate on improving sustainability of global food systems (Hunter et al., 2016).

\subsection{Limitations}

Our study was limited to one village's local food system within the Solomon Islands. Data collection occurred during the lean season, limiting our ability to assess the seasonal dietary fluctuations throughout the year. Our dietary assessments were only conducted with the primary cook from each household and do not represent the intakes of the entire household. However, women of reproductive age are often the most nutritionally vulnerable within a household. Additionally, the nutrient composition data of 53 local food varieties were not available and were therefore substituted with the closest documented variety available. Thus, nutrient variations (positive or negative) were not adequately represented in the diet analysis. Hundreds of PSIDS food varieties are missing nutrient composition databases, leaving their unique nutrient contributions to the diet unknown. Updating the Pacific Island 
Nutrient Composition Tables Version 2 (Dignan et al., 2004), would improve the quality of analysis and agri-food based interventions. These data can also guide policymakers, researchers, and farmers towards cultivate varieties rich in missing essential nutrients as a food-based approach to mitigating malnutrition (Nesbitt et al., 2010), as well an provide opportunities to update nutrition education materials used in this region to provide specific nutritional information regarding the local agrobiodiversity available.

Scientists have documented the dietary changes occurring in the Pacific and anticipated risks nearly 40 years ago (Fitzroy, 1981). However due to lack of historical data and repeated dietary intakes, we are unable to say empirically what the impact of the transforming food system has had on the diets. Our qualitative data provide some insights into the community's perception, opinions and relayed experiences regarding the shift away from traditional agrobiodiverse foods to more modern, imported and processed foods. Villagers shared their concerns with the noticeable rise in T2DM, high blood pressure, and heart disease within their communities, which were previously rare. Further research carried out over longer periods of time at 1- or 2-year intervals over a minimum period of 5 years will allow an exploration on how diets and health outcomes change.

More studies are needed linking agrobiodiversity, nutrition and food systems, especially those that represent additional ecoregions in Solomon Islands. Currently, there is little nationally or regionally representative data that examines Indigenous peoples' local food systems, utilization of agrobiodiversity, diet quality, and food security. The Pacific is extremely data poor, especially regarding food and nutrition security data. More data will provide the necessary groundwork to improve policies, programmes, and educational interventions aimed at achieving zero hunger through the promotion of sustainable food systems.

\section{Conclusion}

Indigenous Solomon Islanders living in Baniata have access to an abundance of healthy agrobiodiverse foods, yet are experiencing a nutrition transition towards a more homogenized western-style dietary pattern. Our findings show an inverse relationship between the number of unique species consumed and body fat percentage. Leveraging agrobiodiversity offers opportunities to improve health outcomes and enhance diets with missing essential nutrients. Encouraging domestic production and consumption of agrobiodiversity also supports local economies and builds foods sovereignty, which is of increasing importance under the threat of global food system disruptions related to climate change.

However, significant tradeoffs exist, as imported and modern foods are often less expensive, more accessible, and quicker to prepare. There is a need for a coordinated nutrition strategy which incorporates the multiple dimensions of sustainable food systems, including nutritional adequacy, sociocultural considerations, cost and economic opportunities, and environmental protection. Through nutrition education, policies, and technological innovations, agrobiodiversity can help support food system resiliency while providing economic opportunities that encourage healthy, balanced, and culturallysignificant dietary patterns.

Supplementary Information The online version contains supplementary material available at https://doi.org/10.1007/s12571-021-01167-7.

Acknowledgements This research would not be possible without the hospitality and participation from the Indigenous villagers from Baniata. In particular, the support both technically and logistically from Manoka Havea, Village Elder from Baniata made this research feasible. Appreciation is given to Solomon Islands National University for providing in-country support, including Carol Offer, Joe Hagabore, and Josephine Maelaua. The Food and Agriculture Organization (FAO) of the United Nations, Agriculture for Nutrition and Health (A4NH) CGIAR Research Programme (CRP) and Massey University provided funding for this research.

Author contributions $\mathrm{CV}, \mathrm{JR}$, and $\mathrm{BB}$ conceptualized the study. $\mathrm{CV}$ and JR ran data analysis. CV and JR interpreted data. CV and JR prepared the draft manuscript. All authors were responsible for contributing to and reviewing the manuscript. CV and JR contributed equally to this article.

\section{Declarations}

Conflict of interest The authors declared that they have no conflict of interest.

Open Access This article is licensed under a Creative Commons Attribution 4.0 International License, which permits use, sharing, adaptation, distribution and reproduction in any medium or format, as long as you give appropriate credit to the original author(s) and the source, provide a link to the Creative Commons licence, and indicate if changes were made. The images or other third party material in this article are included in the article's Creative Commons licence, unless indicated otherwise in a credit line to the material. If material is not included in the article's Creative Commons licence and your intended use is not permitted by statutory regulation or exceeds the permitted use, you will need to obtain permission directly from the copyright holder. To view a copy of this licence, visit http://creativecommons.org/licenses/by/4.0/.

\section{References}

Afshin, A., Sur, P., Fay, K., Cornaby, L., Ferrara, G., Salama, J., Mullany, E., Abate, K., Abbafati, C., Abebe, Z., Afarideh, M., Aggarwal, A., Agrawal, S., Akinyemiju, T., Alahdab, F., Bacha, U., Bachman, V., Badali, H., Badaw, A., et al. (2019). Health effects of dietary risks in 195 countries, 1990-2017: A systematic analysis for the global burden of disease study 2017. The Lancet, 393(10184), 1958-1972.

Allen, M. G. (2015). Framing food security in the Pacific Islands: Empirical evidence from an island in the Western Pacific. Regional Environmental Change, 15(7), 1341-1353. 
Asch, R. G., Cheung, W. W. L., \& Reygondeau, G. (2018). Future marine ecosystem drivers, biodiversity, and fisheries maximum catch potential in Pacific Island countries and territories under climate change. Marine Policy, 88, 285-294.

Asghari, G., Mirmiran, P., Yuzbashian, E., \& Azizi, F. (2017). A systematic review of diet quality indices in relation to obesity. British Journal of Nutrition, 117(8), 1055-1065.

Baker, P., \& Friel, S. (2016). Food systems transformations, ultraprocessed food markets and the nutrition transition in Asia. Globalization and Health, 12(1), 1-15.

Baldermann, S., Blagojević, L., Frede, K., Klopsch, R., Neugart, S., Neumann, A., Ngwene, J., Norkeweit, D., Schröter, A., Schröter, F. J., Wiesner, M., \& Schreiner, M. (2016). Are neglected plants the food for the future? Critical Reviews in Plant Sciences, 35(2), 106-119.

Bekhet, A. K., \& Zauszniewski, J. A. (2012). Methodological triangulation: An approach to understanding data. Nurse Researcher, 20(2), 40-43.

Bell, J., Allain, V., Allison, E. H., Andréfouët, S., Andrew, N. L., Batty, M. J., Blanc, M., Dambacher, J. M., Hampton, J., Hanich, Q., Harley, S., Lorrain, A., McCoy, M., McTurk, N., Nicol, S., Pilling, G., Point, D., Sharp, M. K., Vivili, P., \& Williams, P. (2015). Diversifying the use of tuna to improve food security and public health in Pacific Island countries and territories. Marine Policy, 51, 584-591.

Bell, J. D., Cisneros-Montemayor, A., Hanich, Q., Johnson, J. E., Lehodey, P., Moore, B. R., Pratchett, M. S., Reygondeau, G., Senina, I., Virdin, J., \& Wabnitz, C. C. (2018). Adaptations to maintain the contributions of small-scale fisheries to food security in the Pacific Islands. Marine Policy, 88, 303-314.

Bell, J. D., Sharp, M. K., Havice, E., Batty, M., Charlton, K. E., Russell, J., Adams, W., Azmi, K., Romeo, A., Wabnitz, C. C. C., Andrew, N. L., Rodwell, L., Gu'urau, S., \& Gillett, R. (2019). Realising the food security benefits of canned fish for Pacific Island countries. Marine Policy, 100, 183-191.

Berry, E. M., Dernini, S., Burlingame, B., Meybeck, A., \& Conforti, P. (2015). Food security and sustainability: Can one exist without the other? Public Health Nutrition, 18(13), 2293-2302.

Black, A. (2000). Critical evaluation of energy intake using the Goldberg cut-off for energy intake:Basal metabolic rate. A practical guide to its calculation, use and limitations. International Journal of Obesity, 24(9), 1119-1130

Buah, S., Mlalazi, B., Khanna, H., Dale, J. L., \& Mortimer, C. L. (2016). The quest for Golden bananas: Investigating carotenoid regulation in a Fe'i group Musa cultivar. Journal of Agricultural and Food Chemistry, 64(16), 3176-3185.

Burlingame, B., \& Dernini, S. (2012). Sustainable diets and biodiversity: Directions and solutions for policy, research and action. FAO Headquarters, Rome.

Butte, N. F., \& King, J. C. (2005). Energy requirements during pregnancy and lactation. Public Health Nutrition, 8(7a), 1010-1027.

Canavan, C. R., Noor, R. A., Golden, C. D., Juma, C., \& Fawzi, W. (2017). Sustainable food systems for optimal planetary health. Transactions of the Royal Society of Tropical Medicine and Hygiene, 111(6), 238-240.

CDC. (2011). Anthropometry Procedures Manual. Retrieved 8 January 2020, from https://www.cdc.gov/nchs/data/nhanes/nhanes_11_12/ Anthropometry_Procedures_Manual.pdf

Charrondiere, U, R., Haytowitz, D., \& Stadlmayr, B. (2012). FAO/ INFOODS density database version 2.0. Fao/Infoods, 2, 24. Retrieved 8 January 2020, from http://www.fao.org/docrep/017/ ap815e/ap815e.pdf

Chase, L., \& Grubinger, V. (2014). Food, farms, and community: Exploring food systems. University of New Hampshire Press.

Convention on Biological Diversity, FAO, The World Bank, UNEP, \& UNDP. (2015). Biodiversity and the 2030 Agenda for Sustainable Development. Retrieved 12 Feburary 2020, from https://www.cbd.int/ development/doc/biodiversity-2030-agenda-technical-note-en.pdf
Dignan, C., Burlingame, B., Shailesh, K., \& Aalbersberg, W. (2004). The Pacific Islands food composition tables second edition. FAO.

Dweba, T. P., \& Mearns, M. A. (2011). Conserving indigenous knowledge as the key to the current and future use of traditional vegetables. International Journal of Information Management, 31(6), 564-571.

Ebert, A. W. (2014). Potential of underutilized traditional vegetables and legume crops to contribute to food and nutritional security, income and more sustainable production systems. Sustainability (Switzerland), 6(1), 319-335.

FAO. (2014). Guidelines for assessing nutrition-related Knowledge, Attitudes and Practices manual. Retrieved April 12, 2019, from http://www.fao.org/3/a-i3545e.pdf

FAO. (2016). Minimum dietary diversity for women- a guide to measurement. Minimum Dietary Diversity for Women: A Guide for Measurement. Retrieved April 12, 2019, from http://www.fao.org/ 3/i5486e/i5486e.pdf

FAO. (2017). The Food Insecurity Experience Scale: Measuring food insecurity through people's experiences. Retrieved June 20, 2019, from http://www.fao.org/3/a-i7835e.pdf

FAO. (2018). FAO/INFOODS Food Composition Databases. Retrieved May 6, 2019, from http://www.fao.org/infoods/infoods/tables-anddatabases/faoinfoods-databases/en/

FAO. (2019). FAOSTAT Food Balance Sheets. Retrieved July 16, 2019, from http://www.fao.org/faostat/en/\#data/FBS

FAO; WHO. (2004). Fruits and Vegetables for Health: Report of a Joint FAO/WHO Workshop. Retrieved April 30, 2019, from https://apps. who.int/iris/handle/10665/43143

FAO/WHO. (2010). Fats and fatty acids in human nutrition. Retrieved July 16, 2019, from http://agris.fao.org/agris-search/search.do? recordID $=$ XF2016049106

FAO-Stat. (2018). FAO Country Profile - Solomon Islands. Retrieved April 30, 2019, from http://www.fao.org/countryprofiles/index/ en/?iso3=SLB

Fitzroy, J. (1981). Influence of development factors on nutritional patterns in the Solomon Islands. Ecology of Food and Nutrition, 10(3), 187-191.

Foley, J. A., Ramankutty, N., Brauman, K. A., Cassidy, E. S., Gerber, J. S., Johnston, M., Mueller, N. D., O'Connell, C., Ray, D. K., West, P. C., Balzer, C., Bennett, E. M., Carpenter, S. R., Hill, J., Monfreda, C., Polasky, S., Rockström, J., Sheehan, J., Siebert, S., Tilman, D., \& Zaks, D. P. M. (2011). Solutions for a cultivated planet. Nature, 478(7369), 337-342.

Friel, S., Chopra, M., \& Satcher, D. (2007). Unequal weight: Equity oriented policy responses to the global obesity epidemic. BMJ, 335(7632), 1241-1243.

Fujinuma, R., Kirchhof, G., Ramakrishna, A., Sirabis, W., Yapo, J., Woruba, D., Gurr, G., \& Menzies, N. (2018). Intensified sweetpotato production in Papua New Guinea drives plant nutrient decline over the last decade. Agriculture, Ecosystems and Environment, 254, 10-19.

Gibson, R. S., \& Cavalli-Sforza, T. (2012). Using reference nutrient density goals with food balance sheet data to identify likely micronutrient deficits for fortification planning in countries in the Western Pacific region. Food and Nutrition Bulletin, 33(3 Suppl), 214-220.

Gibson, R. S., Charrondiere, U. R., \& Bell, W. (2017). Measurement errors in dietary assessment using self-reported 24-hour recalls in low-income countries and strategies for their prevention. Advances in Nutrition: An International Review Journal, 8(6), 980-991.

Granderson, A. (2017). The role of traditional knowledge in building adaptive capacity for climate change: Perspectives from Vanuatu. Weather, Climate, and Society, 9(3), 545-561.

Haddad, L., Cameron, L., \& Barnett, I. (2015). The double burden of malnutrition in SE Asia and the Pacific: Priorities, policies and politics. Health Policy and Planning, 30(9), 1193-1206. 
Hariri, N., Gougeon, R., \& Thibault, L. (2010). A highly saturated fat-rich diet is more obesogenic than diets with lower saturated fat content. Nutrition Research, 30(9), 632-643.

Harttig, U., Haubrock, J., Knüppel, S., \& Boeing, H. (2011). The MSM program: Web-based statistics package for estimating usual dietary intake using the multiple source method. European Journal of Clinical Nutrition, 65(S1), S87-S91.

Haynes, E., Brown, C. R., Wou, C., Vogliano, C., Guell, C., \& Unwin, N. (2018). Health and other impacts of community food production in Small Island developing states: A systematic scoping review. Revista Panamericana de Salud Publica, Pan American Journal of Public Health, 42, e176.

He, K., Hu, F. B., Colditz, G. A., Manson, J. E., Willett, W. C., \& Liu, S. (2004). Changes in intake of fruits and vegetables in relation to risk of obesity and weight gain among middle-aged women. International Journal of Obesity, 28(12), 1569-1574.

Headey, D., Hirvonen, K., \& Hoddinott, J. (2018). Animal sourced foods and child stunting. American Journal of Agricultural Economics, 100(5), 1302-1319.

Hodgson, J. M., Hsu-Hage, B. H., \& Wahlqvist, M. L. (1994). Food variety as a quantitative descriptor of food intake. Ecology of Food and Nutrition, 32(3-4), 137-148.

Hooper, L., Abdelhamid, A., Bunn, D., Brown, T., Summerbell, C. D., \& Skeaff, C. M. (2015). Effects of total fat intake on body weight. Cochrane Database of Systematic Reviews, 8.

Horsey, B., Swanepoel, L., Underhill, S., Aliakbari, J., \& Burkhart, S. (2019). Dietary diversity of an adult Solomon Islands population. Nutrients, 11(7), 1622.

Hughes, R. G., \& Lawrence, M. A. (2005). Globalisation, food and health in Pacific Island countries. Asia Pacific Journal of Clinical Nutrition, 14(April), 298-306.

Hunter, D., Özkan, I., de Oliveira Beltrame, D., Samarasinghe, W. L. G., Wasike, V. W., Charrondière, U. R., Borelli, T., \& Sokolow, J. (2016). Enabled or disabled: Is the environment right for using biodiversity to improve nutrition? Frontiers in Nutrition, 3, 14.

Jayawardena, R., Byrne, N. M., Soares, M. J., Katulanda, P., Yadav, B., \& Hills, A. P. (2013). High dietary diversity is associated with obesity in Sri Lankan adults: An evaluation of three dietary scores. $B M C$ Public Health, 13(1), 1-8.

Konishi, S., Watanabe, C., Umezaki, M., \& Ohtsuka, R. (2011). Energy and nutrient intake of Tongan adults estimated by 24-hour recall: The importance of local food items. Ecology of Food and Nutrition, 50(4), 337-350.

Lachat, C., Raneri, J. E., Smith, K. W., Kolsteren, P., Van Damme, P., Verzelen, K., Penafiel, D., Vanhove, W., Kennedy, G., Hunter, D., Odhiambo, F. O., Ntandou-Bouzitou, G., Baets, B. E., Ratnasekera, D., Ky, H. T., Remans, R., \& Termote, C. (2018). Dietary species richness as a measure of food biodiversity and nutritional quality of diets. Proceedings of the National Academy of Sciences of the United States of America, 115(1), 127-132.

Lee, C. M. Y., Colagiuri, S., Ezzati, M., \& Woodward, M. (2011). The burden of cardiovascular disease associated with high body mass index in the Asia-Pacific region. Obesity Reviews, 12(5), e454- e459.

Li, X., \& Siddique, K, H, M. (2018). Future Smart Foods: Rediscovering hidden treasures of neglected and underutilized species for Zero Hunger in Asia. Retrieved January 21, 2019, from http://www.fao. org/3/I8907EN/i8907en.pdf

Lin, S.-H., Lin, Y.-F., Cheema-Dhadli, S., Davids, M. R., \& Halperin, M. L. (2002). Hypercalcaemia and metabolic alkalosis with betel nut chewing: Emphasis on its integrative pathophysiology. Nephrology Dialysis Transplantation, 17(5), 708-714.

Lin, T. K., Teymourian, Y., \& Tursini, M. S. (2018). The effect of sugar and processed food imports on the prevalence of overweight and obesity in 172 countries. Globalization and Health, 14(1), 35.

Masset, G., Soler, L. G., Vieux, F., \& Darmon, N. (2014). Identifying sustainable foods: the relationship between environmental impact, nutritional quality, and prices of foods representative of the French diet. Journal of the Academy of Nutrition and Dietetics, 114(6), 862-869.

McIver, L., Kim, R., Woodward, A., Hales, S., Spickett, J., Katscherian, D., Hashizume, M., Honda, Y., Kim, H., Iddings, S., Naicker, J., Bambrick, H., McMichael, A. J., \& Ebi, K. L. (2016). Health impacts of climate change in Pacific Island countries: A regional assessment of vulnerabilities and adaptation priorities. Environmental Health Perspectives, 124(11), 1707-1714.

McLennan, A. K., \& Ulijaszek, S. J. (2015). Obesity emergence in the Pacific islands: Why understanding colonial history and social change is important. Public Health Nutrition, 18(8), 1499-1505.

Michou, M., Panagiotakos, D. B., \& Costarelli, V. (2018). Low health literacy and excess body weight: A systematic review. Central European Journal of Public Health, 26(3), 234-241.

Ministry of Health and Medical Services. (2017). Solomon Islands National NCD Action Plan A Multi-sectoral Approach to Prevent Lifestyle-Related Diseases. Retrieved January 30, from https:// extranet.who.int/nutrition/gina/sites/default/files/SLB-2010-2017NCD_0.pdf

Mohee, R., Mauthoor, S., Bundhoo, Z. M. A., Somaroo, G., Soobhany, N., \& Gunasee, S. (2015). Current status of solid waste management in small island developing states: A review. Waste Management, 43, 539-549.

Monteiro, C. A., Cannon, G., Levy, R. B., Moubarac, J.-C., Louzada, M. L., Rauber, F., Khandpur, N., Cediel, G., Neri, D., Martinez-Steele, E., Baraldi, L. G., \& Jaime, P. C. (2019). Ultra-processed foods: What they are and how to identify them. Public Health Nutrition, 22(5), 936-941.

Moubarac, J.-C., Batal, M., Louzada, M. L., Martinez Steele, E., \& Monteiro, C. A. (2017). Consumption of ultra-processed foods predicts diet quality in Canada. Appetite, 108, 512-520.

National Academies Press. (2000). Using the Estimated Average Requirement for Nutrient Assessment of Groups. Retrieved September 20, 2019, from https://www.ncbi.nlm.nih.gov/books/ NBK222898/

Neelakantan, N., Seah, J. Y. H., \& van Dam, R. M. (2020). The effect of coconut oil consumption on cardiovascular risk factors: A systematic review and meta-analysis of clinical trials. Circulation, 141(10), 803-814.

Nesbitt, M., McBurney, R. P. H., Broin, M., \& Beentje, H. J. (2010). Linking biodiversity, food and nutrition: The importance of plant identification and nomenclature. Journal of Food Composition and Analysis, 23(6), 486-498.

Nilles, E. J., Manaia, A., Ruaia, B., Huppatz, C., Ward, C., George, P., Sies, C., Cangiano, A., Sejvar, J., \& Tira, T. (2018). Re-emergence of thiamine deficiency disease in the Pacific islands (2014-15): A case-control study. PLoS One, 13(6), e0198590.

Nishida, C., Ko, G. T., \& Kumanyika, S. (2010). Body fat distribution and noncommunicable diseases in populations: Overview of the 2008 WHO expert consultation on waist circumference and waisthip ratio. European Journal of Clinical Nutrition, 64(1), 2-5.

Pacific Adaptation Strategy Assistance Program. (2012). Roviana Climate Change Resilience Plan 2013-2017. Retrieved April 21, 2019, from https://www.environment.gov.au/system/files/pages/ f7e2f421-2423-40a3-8842-85a89805cb0a/files/roviana-climatechange-resilience-plan-2013-2017.pdf

Padhi, M. (2016). Importance of indigenous breeds of chicken for rural economy and their improvements for higher production performance. Hindawi Publishing Corporation. https://doi.org/10.1155/ $2016 / 2604685$

Phalkey, R. K., Aranda-Jan, C., Marx, S., Höfle, B., \& Sauerborn, R. (2015). Systematic review of current efforts to quantify the impacts of climate change on undernutrition. Proceedings of the National Academy of Sciences of the United States of America, 112(33), E4522-E4529. 
Pollock, N. J. (2017). Diversification of foods and their values: Pacific foodscapes. In Tides of innovation in Oceania: Value, materiality and place. ANU Press.

Popkin, B. M. (2015). Nutrition transition and the global diabetes epidemic. Current diabetes reports, 15(9), 1-8.

Popkin, B. M., \& Gordon-Larsen, P. (2004). The nutrition transition: Worldwide obesity dynamics and their determinants. International Journal of Obesity, 28, S2-S9.

Poti, J. M., Braga, B., \& Qin, B. (2017). Ultra-processed food intake and obesity: What really matters for health-Processing or nutrient content? Current Obesity Reports, 6(4), 420-431.

Raneri, J. E., Padulosi, S., Meldrum, G., \& King, O. I. (2019). Promoting neglected and underutilized species to boost nutrition in LMICs. UNSCN Nutrition, 44, 10-25.

Rischkowsky, B., \& Pilling, D. (2007). The state of the World's animal genetic resources for food and agriculture. FAO. Retrieved April 21, 2019, from: http://www.fao.org/3/a1260e/a1260e.pdf.

Roos, C. I., Field, J. S., \& Dudgeon, J. V. (2016). Anthropogenic burning, agricultural intensification, and landscape transformation in postLapita Fiji. Journal of Ethnobiology, 36(3), 535-553.

Roza, A. M., \& Shizgal, H. M. (1984). The Harris Benedict equation reevaluated: Resting energy requirements and the body cell mass. The American Journal of Clinical Nutrition, 40(1), 168-182.

Sandifer, P. A., Sutton-Grier, A. E., \& Ward, B. P. (2015). Exploring connections among nature, biodiversity, ecosystem services, and human health and well-being: Opportunities to enhance health and biodiversity conservation. Ecosystem Services, 12, 1-15.

Santos, J. A., McKenzie, B., Trieu, K., Farnbach, S., Johnson, C., Schultz, J., Thow, A. M., Snowdon, W., Bell, C., \& Webster, J. (2019). Contribution of fat, sugar and salt to diets in the Pacific Islands: A systematic review. Public Health Nutrition, 22(10), 1858-1871.

Shrimpton, R., Mbuya, N, V., \& Provo, A, M. (2016). The double burden of malnutrition in East Asia and the Pacific. Retrieved December 21, 2018, from https://openknowledge.worldbank.org/handle/10986/26102.

Sievert, K., Lawrence, M., Naika, A., \& Baker, P. (2019). Processed foods and nutrition transition in the Pacific: Regional trends, patterns and food system drivers. Nutrients 2019, 11(6), 1328.

Smith, P., \& Gregory, P. J. (2013). Climate change and sustainable food production. The Proceedings of the Nutrition Society, 72(1), 21-28.

Smith, M. R., \& Myers, S. S. (2018). Impact of anthropogenic CO2 emissions on global human nutrition. Nature Climate Change, $8(9), 834-839$

Smith, M. D., Kassa, W., \& Winters, P. (2017). Assessing food insecurity in Latin America and the Caribbean using FAO's food insecurity experience scale. Food Policy, 71, 48-61.
Snowdon, W., Raj, A., Reeve, E., Guerrero, R. L., Fesaitu, J., Cateine, K., \& Guignet, C. (2013). Processed foods available in the Pacific Islands. Globalization and Health, 9(1), 53.

Solomon Islands MET. (2011). Current and future climate of the Solomon Islands South Pacific Ocean Solomon Sea. Retrieved March 23, 2019, from https://www.pacificclimatechangescience.org/wp-content/ uploads/2013/06/13_PCCSP_Solomon_Islands_8pp.pdf

Solomon Islands National Statistics Office. (2015). Solomon Islands 2012/12 Household Income and Expenditure Survey: Provincial Analytical Report (Volume II). Retrieved March 3, 2019, from https://www.statistics.gov.sb/component/advlisting/?view= download\&format=raw\&fileId $=409$

Taylor, M. K., Sullivan, D. K., Ellerbeck, E. F., Gajewski, B. J., \& Gibbs, H. D. (2019). Nutrition literacy predicts adherence to healthy/ unhealthy diet patterns in adults with a nutrition-related chronic condition. Public Health Nutrition, 22(12), 2157-2169.

The Lancet. (2019). Saving the Pacific islands from extinction. The Lancet., 394(10196), 359.

Thow, A. M., Heywood, P., Schultz, J., Quested, C., Jan, S., \& Colagiuri, S. (2011). Trade and the nutrition transition: Strengthening policy for health in the pacific. Ecology of Food and Nutrition, 50(1), 18-42.

UNDESA. (2012). World Population Prospects: Population Division Database. Retrieved June 20, 2019, from http://hdr.undp.org/en/data

UNICEF/WHO. (2019). Solomon Island Statistics. Retrieved March 28, 2019, from https://www.unicef.org/infobycountry/solomonislands statistics.html

Warrick, O., Aalbersberg, W., Dumaru, P., McNaught, R., \& Teperman, K. (2017). The 'Pacific adaptive capacity analysis framework': Guiding the assessment of adaptive capacity in Pacific island communities. Regional Environmental Change, 17(4), 1039-1051.

Wheeler, T., \& von Braun, J. (2013). Climate change impacts on global food security. Science, 341(6145), 508-513.

WHO. (2012). Sodium intake for adults and children. Retrieved April 21, 2018, from https://apps.who.int/iris/bitstream/handle/10665/77985/ 9789241504836_eng.pdf

WHO \& FAO. (2004). Vitamin and mineral requirements in human nutrition. World Health Organization.

Zimmerer, K. S., \& De Haan, S. (2017). Agrobiodiversity and a sustainable food future. Nature Plants, 3(4), 1-3.

Zimmerer, K. S., de Haan, S., Jones, A. D., Creed-Kanashiro, H., Tello, M., Carrasco, M., Meza, K., Amaya, F. P., Cruz-Garcia, G. S., Tubbeh, R., \& Jiménez Olivencia, Y. (2019). The biodiversity of food and agriculture in the anthropocene: Research advances and conceptual framework. Anthropocene, 25, 100192. 


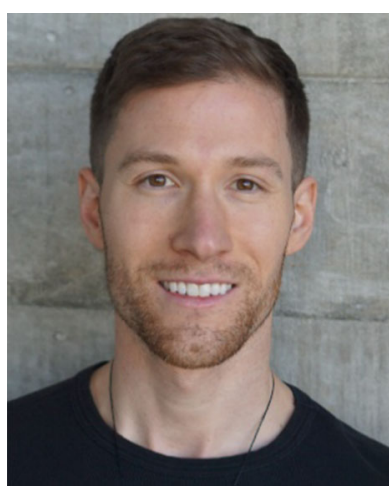

Chris Vogliano MS, RD is a research fellow for Bioversity International working on a $\mathrm{FAO} /$ $\mathrm{A} 4 \mathrm{NH}$ funded research project in the Solomon Islands looking at the role of biodiversity in indigenous food systems and diet quality. Chris is a public health dietitian who has expertise in alleviating food insecurity and promoting sustainable diets through educational programming, policy interventions, and research. Chris has served as an agriculture, nutrition and health research fellow for The Academy of Nutrition and Dietetics Foundation, was awarded the "Young Dietitian of the Year" by the state of Washington, and is currently pursuing his Ph.D. at Massey University in Wellington, New Zealand, focused on promoting biodiverse and sustainable food systems among Pacific Small Island Developing States (PSIDS).

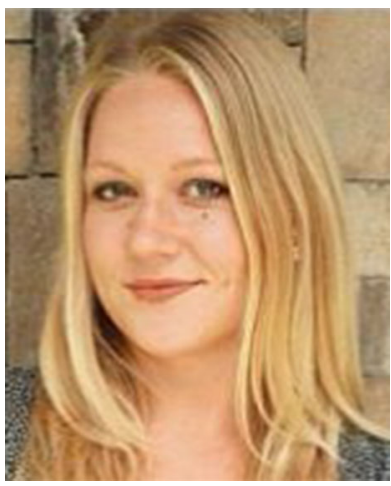

Jessica Raneri is a Nutrition Research Specialist involved in designing and implementing 'Agricultural Biodiversity, Nutrition and Dietary' assessments using participatory, qualitative and quantitative methods and has over 7 years of experience working in nutrition-sensitive agriculture in the Asia Pacific Region. Jessica has been providing technical support to guide agricultural investments to have food system resilience and nutrition sensitive lenses, to facilitate achieving nutrition and climate resilience outcomes. She has led numerous research programs that focus on implementing nutrition packages that promote nutrition education and diversification of local food systems (including homestead production systems and markets) with nutritious foods to enable households to directly access these foods (food gardens), as well as utilize income generated from the value chain projects to reinvest into their household's nutrition (market diversity).

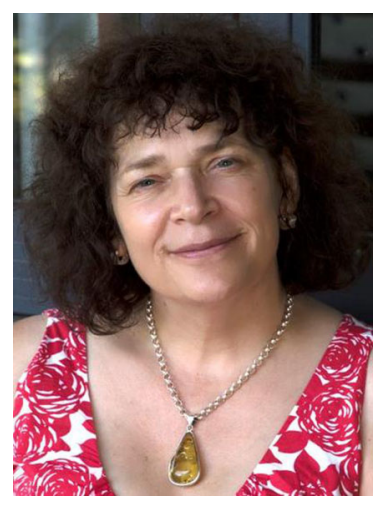

Jane Coad is a Professor in Nutrition at Massey University under the School of Food and Advanced Technology. Jane's research interests include micronutrient deficiencies, diabetes mellitus type 2 , IBD, and maternal and infant nutrition.

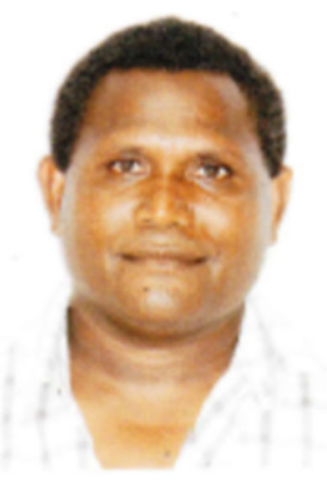

Shane started his career as a lecturer at the School of Natural Resources, Solomon Islands College of Higher Education (now Solomon Islands National University), teaching soils and environmental sciences to tropical agriculture and forestry students. His interests include nutrition sensitive agriculture, agrobiodiversity and sustainable production systems such as agroforestry and organic farming systems and how they influence soil health and adapt to climate change. Shane holds a Masters in soil chemistry and biology from Lincoln University, New Zealand, and a PhD on the biogeochemistry of soil carbon and nutrient cycling in forest plantations from Griffith University, Australia. He is currently managing Zai Na Tina Centre for Organic Systems, which operates an organic farm and provides practical training and research on organics at the outskirts of Honiara, Solomon Islands. He also operates SPE Analytical \& Scientific, a private laboratory in Honiara, and co-owns SolAgro, which value adds and trades the ngali nut, an indigenous nut in Solomon Islands. Shane lives with his wife, Mishella, and two sons, Talena and Irava, at Zai Na Tina Organic Farm where they grow their own organic vegetables and enjoy the farm view from their balcony. 


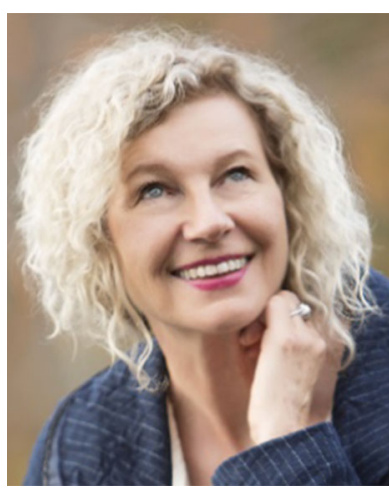

Carol Wham is an associate professor of nutrition and dietetics at Massey University. As the public health nutrition domain leader in the Nutrition and Dietetics programme her research interests include diet and lifestyle interventions across the life span. Currently her main research focus is the relationship between nutrition status and health outcomes in the ageing population. She has been researching this topic within two longitudinal studies of ageing and by measuring risk factors associated with malnutrition among older people in various accommodation settings.

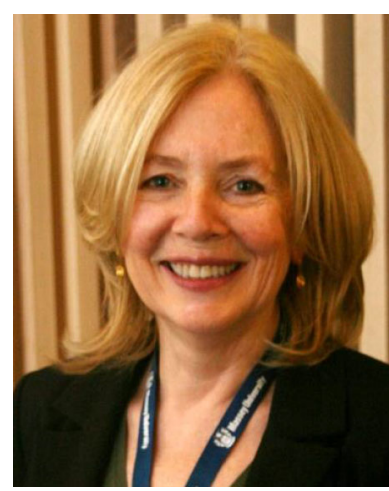

Barbara Burlingame is a Professor of Nutrition and Food Systems and former nutrition chief with the UN's FAO. Her roles include that of chair/ scientific adviser/board member of several foundations/academies, editor of Frontiers in Nutrition a n d Environ m e n t a 1 Sustainability, and several adjunct professorships. She's author/ editor of hundreds of papers, books, book chapters, UN reports and policy documents in the areas biodiversity for food and nutrition, sustainable diets, indigenous food systems, and food composition.

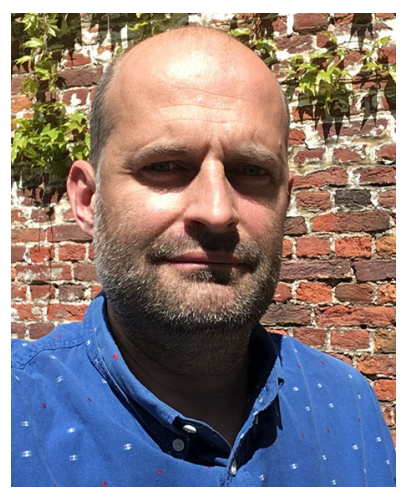

Carl Lachat is a Professor at Ghent University with the Department of Food Technology, Safety and Health, and lectures in food and nutrition epidemiology, participatory planning and evaluation, nutrition interventions and nutrition policies. He has over 15 years' experience working in academia with interests focused around nutrition research methodologies, agrobiodiversity and nutrition and sustainable diets. 